\title{
Exploiting the overhearing capabilities of transmitting nodes to increase the energy efficiency in dense networks
}

\author{
Rodolfo Torrea-Duran ${ }^{1 *}$ (D, Fernando Rosas ${ }^{2}$, Sofie Pollin ${ }^{3}$, Luc Vandendorpe ${ }^{4}$ and Marc Moonen ${ }^{1}$
}

\begin{abstract}
The 1000-fold capacity increase envisioned by dense $5 \mathrm{G}$ networks results also in a tremendous increase in the energy consumption of the whole network. Utilizing relays in combination with physical-layer network coding (PNC) has been proposed as an energy-efficient solution to this problem by creating several short-distance low-power transmissions and by reducing the transmission time. However, deploying relay nodes can be very costly for dense networks. On the other hand, the proximity of transmitting nodes in dense networks allows the transmitting nodes to serve as relays and retransmit the signals overheard from other transmitting nodes using PNC. This approach has been shown to increase the spectral efficiency, but the impact on energy efficiency has not been studied yet. Therefore, in this paper, we analyze two approaches that exploit the overhearing capabilities of the transmitting nodes in terms of spectral efficiency, energy efficiency, and success rate. We then provide a low-complexity power control strategy that achieves a performance close to the optimal for each approach. We show that when at least one indirect link is stronger than the direct links, exploiting the overhearing capabilities of the transmitting nodes provides the highest performance in both the transmit power-dominated and circuit power-dominated regimes.
\end{abstract}

Keywords: Energy efficiency, Relaying, Overhearing capabilities, Dense networks

\section{Introduction}

5G wireless networks aim for a 1000-fold increase of the system capacity compared to current state-of-the-art technology [1]. To achieve this, a tremendous increase in the number of base stations and connected devices is expected in the coming years, increasing not only the density of the network [2,3] but also its energy consumption. Therefore, finding ways to increase the energy efficiency while maintaining a high spectral efficiency is one of the main challenges in wireless networks for a sustainable growth, both economically and environmentally [4].

Relays have been proposed to deal with these challenges by dividing long-distance transmissions into several short-distance low-power transmissions $[5,6]$.

\footnotetext{
*Correspondence: Rodolfo.TorreaDuran@esat.kuleuven.be

'STADIUS Center for Dynamical Systems, Signal Processing and Data Analytics, Department of Electrical Engineering (ESAT), KU Leuven, Kasteelpark Arenberg 10, 3000 Leuven, Belgium

Full list of author information is available at the end of the article
}

However, relay nodes require certain coordination with the rest of the network nodes in order to manage transmissions efficiently. Cooperative protocols have thus gained much attention by using relays to retransmit the overheard information to an intended receiver.

One of these protocols combines relays with physicallayer network coding (PNC) $[7,8]$. Much of the available literature about relaying schemes using PNC focuses on the particular case of the two-way relay channel (TWRC) with the goal of increasing the achievable data rate [9-11]. Moreover, the works that study the energy efficiency of relaying schemes using PNC are also limited to the TWRC case. For instance, the authors in [12] propose an optimal power allocation strategy for a TWRC using PNC with per-node power constraints, while the authors in [13] use end-to-end rate constraints. In [14], the authors show that TWRC transmissions are more energy efficient in the high spectral efficiency and large path loss attenuation region compared to direct 
transmissions and one-way relay transmissions. In [6], the authors show that TWRC transmissions are more energy efficient in symmetric systems where the circuit power consumption and the transmitted bits in both directions are equal. Finally, in [15], the energyefficient resource allocation for OFDMA-based two-way relay is studied by maximizing the aggregate energy efficiency while providing proportional fairness in energy efficiency.

A main disadvantage of these approaches is that by considering a TWRC, received signals come only from the relay node, hence disregarding potentially useful signals originating directly from the transmitting node. A more general relay configuration using PNC is proposed in [16], where signals are coming from both the transmitting node and the relay node. This makes more sense in terms of energy efficiency since all the transmitted power can be utilized in some way. However, all the previous approaches require the deployment of relays, which can be costly or even prohibitive for dense networks.

A similar effect can be achieved if, instead of deploying relay nodes, the overhearing capabilities of the transmitting nodes are exploited by allowing these to retransmit the signals overheard from other transmitting nodes. In this way, the transmit power from all the transmitting nodes is utilized, while the deployment of relays is avoided. A main challenge in this case is that the resources need to be split for serving the intended user and other users. Taking this into account, it has been shown that cooperation among transmitting nodes can actually improve the users' energy efficiency $[17,18]$.

Future networks foresee the proliferation of additional base stations (microcells, picocells, femtocells) underlaid in the traditional (macro) cellular network in order to increase the area spectral efficiency [19]. Similar to relays, these base stations can also help to increase the energy efficiency of the network, especially for a high area throughput and a high user density [20]. These additional base stations often do not count with a backhaul link for data transmission, while the backhaul operating costs are substantial [21]. Hence, the potential increase in spectral and energy efficiency of dense networks serves as incentive for the base stations to act as relays. Furthermore, in dense networks, the proximity of the nodes results in a high probability of finding transmitting nodes with a strong link between them as seen in Fig. 1. For instance, neighboring base stations in a dense network can retransmit the overheard signals from the other base stations in order to increase both the downlink data rate and the energy efficiency. Similarly, energy-limited devices in close proximity can retransmit the overheard signals from other devices through a D2D link to increase the total uplink data rate.

A way of exploiting the overhearing capabilities of transmitting nodes using PNC principles is through space-time network coding (STNC) [22]. With STNC, each of the $L$ transmitting nodes transmits its own signal in a dedicated time slot during the transmission phase, and during the relaying phase $M$, relay nodes (or $M$ transmitting nodes that overhear previous transmissions) retransmit

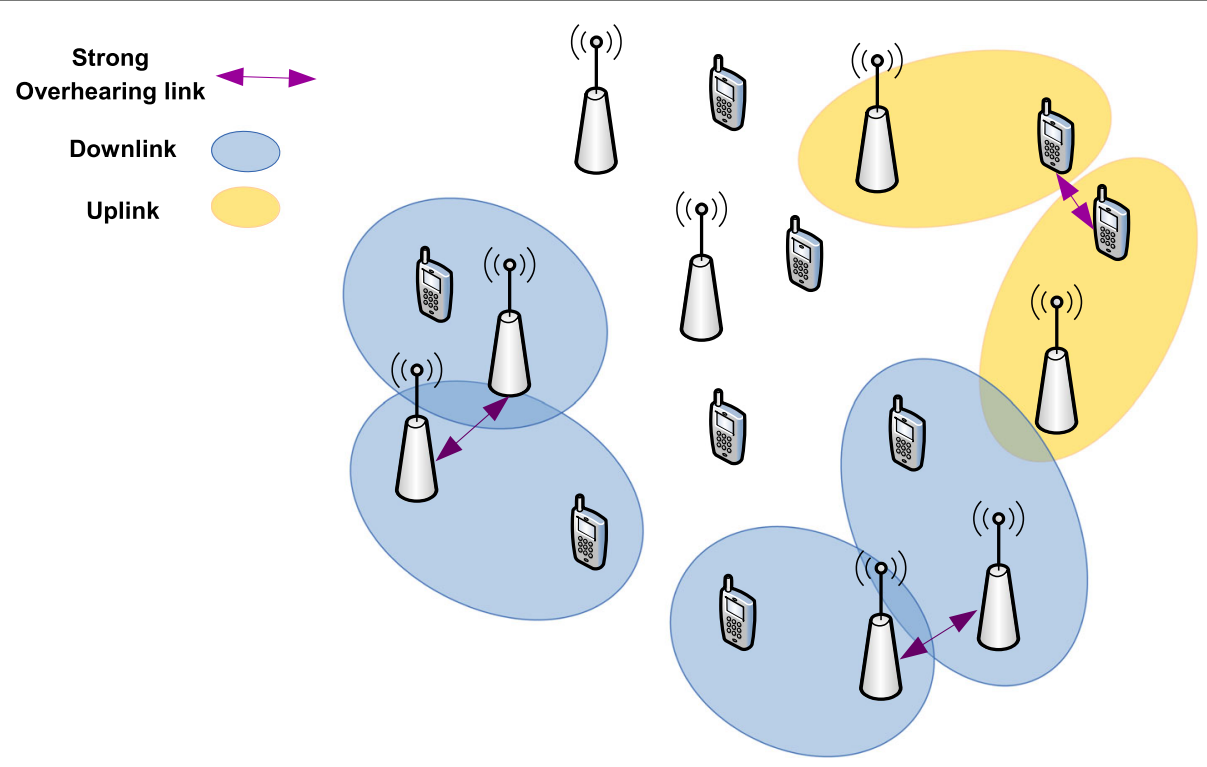

Fig. 1 Exploiting the strong overhearing link in dense networks for downlink and uplink communication 
in dedicated time slots a combination of the signals received in the transmission phase, hence achieving diversity order of $M+1$. A step to increase the diversity of the previous system is taken in [23] and in [24], where the authors propose that each relay decodes the signal not only from the transmitting nodes but also from the previously transmitting relays. However, a main disadvantage of this approach is that it requires $L+M$ time slots. As this number grows with the number of nodes, this approach is difficult to implement in large networks.

An alternative that also exploits the overhearing capabilities of transmitting nodes with fewer time slots is proposed in [25]. Similar to [22-24], each of the $L$ transmitting nodes transmits its own signal in a dedicated time slot during the transmission phase. Then, during the relaying phase, all the transmitting nodes retransmit a combination of the previously overheard signals simultaneously, hence requiring only $L+1$ time slots. Combining transmissions from different transmitting nodes in one time slot can drastically reduce the number of time slots required per transmission round, but it can also increase the interference during the relaying phase.

In terms of data rate, it is clear that when the (direct) link between a transmitter and its intended receiver is strong, relaying might not be necessary. However, when the interference is strong, exploiting alternative (indirect) links proves beneficial, either using a single or dedicated time slots during the relaying phase. However, the energy efficiency analysis is not straightforward since relaying might have a larger delay and energy cost compared to the data rate benefit. Furthermore, the energy efficiency might be very different when the transmit power is the largest energy contributor compared to when the circuit power is the largest contributor. To the best of our knowledge, an energy efficiency analysis of this type of approaches that exploit the overhearing capabilities of the transmitting nodes has not been reported in literature.

The first contribution of this paper consists in the analysis of two approaches (the first one based on [22] and the second one based on [25]) that exploit the overhearing capabilities of transmitting nodes in terms of spectral efficiency, energy efficiency, and success rate. The first approach (referred to as DIV) is a variation of the traditional space-time network coding (STNC) approach that uses no relays but the same transmitting nodes to relay overheard information. The second approach (referred to as DRCP) also exploits the overhearing capabilities of transmitting nodes but uses fewer time slots. The motivation behind this energy efficiency analysis is that both approaches aim to utilize the power transmitted by other nodes. Although we limit the analysis to the case of two transmitting nodes and two receiving nodes, the ideas presented here can also be used in a larger scenario. The second contribution consists in a low-complexity power control strategy that achieves a similar energy efficiency as the optimal strategy for each approach (DIV and DRCP). The proposed strategy requires only a comparison of the channel conditions between direct and indirect links. This results in a closed-form formula for each case. We are then able to show that when at least one indirect link is stronger than the direct links, exploiting the overhearing capabilities of the transmitting nodes provides the highest performance. This is particularly beneficial for dense networks since there is a high probability of finding strong indirect links.

The rest of the paper is organized as follows. Section 2 describes the system model. Section 3 presents the two baseline approaches not exploiting overhearing capabilities. Section 4 presents the energy efficiency optimization of two approaches that exploit overhearing capabilities. Section 5 presents the complexity analysis. Section 6 shows the performance evaluation. Finally, Section 7 draws some conclusions.

\section{System model}

Consider a system composed by a pair of transmitting nodes (TXs), each of which has data to be delivered to a specific receiving node (RX). We assume that all the nodes are half duplex (i.e., they cannot transmit and receive simultaneously) and that no backhaul link exists between the TXs. This last condition excludes the use of transmission schemes that require coordinated transmitters or antennas, e.g., space-time block codes such as Alamouti codes. We also assume that each TX can overhear the transmission of the other TXs in a reliable way, i.e., that the links between TXs (overhearing links) are stronger than the links between TXs and RXs (direct/indirect links).

We consider a standard power model including transmit power and circuit power. While the transmit power is radiated by the TXs for data transmission, the circuit power represents the average energy consumption of electronic components, typically modeled as a constant, independent of the transmission rate [17]. A regime in which the transmit power dominates over the circuit power is typical for cellular networks, where TXs need to compensate for the large path loss of communication over long distances. On the other hand, a regime in which the circuit power dominates over the transmit power corresponds to short-range communication scenarios [26]. In this paper, we focus on the case where both TXs operate either in the transmit power regime or in the circuit power regime. 
Let us denote $s_{l}$ as the symbol from the $l$ th TX to be delivered to the $l$ th RX. Symbols are assumed to be uncorrelated. Let us define $P_{l}^{(t)}$ as the transmit power of TX $l$ in time slot $t$ and $p_{l}^{(t)}$ as the (constant) circuit power of TX $l$ in time slot $t$. We also define $\gamma_{l m}^{(t)}=\frac{\sigma_{s}^{2}}{\sigma_{\mathrm{n}}^{2}} P_{l}^{(t)}\left|h_{l m}\right|^{2}$ and $\xi_{l m}^{(t)}=\frac{\sigma_{\mathrm{s}}^{2}}{\sigma_{\mathrm{n}}^{2}} P_{l}^{(t)}\left|g_{l m}\right|^{2}$, where $h_{l m}$ is the link from TX $l$ to RX $m$ and $g_{l m}$ is the link from TX $l$ to TX $m$ (overhearing link). If $l=m$, we call $h_{l m}$ a direct link, else we call $h_{l m}$ an indirect link. Given the likelihood of finding strong overhearing links in dense networks, in this paper, we focus on the case where the overhearing links are stronger than the other communication links, i.e., $\xi_{l m}^{(t)} \gg$ $\gamma_{m n}^{(t)}, \forall l, m, n$. The parameter $\sigma_{s}^{2}=\mathbb{E}\left\{\left|s_{1}\right|^{2}\right\}=\mathbb{E}\left\{\left|s_{2}\right|^{2}\right\}$, and $\sigma_{n}^{2}$ is the received noise power, assumed to be equal for all nodes. Without loss of generality, we assume $\sigma_{s}^{2}=\sigma_{n}^{2}=1$.

We define the energy efficiency $\epsilon$ as the number of bits received by a RX in units of bits per unit of bandwidth per unit of energy. The energy efficiency can then be expressed as a function of the spectral efficiency (bits per unit of bandwidth per unit of time) and the power (energy per unit of time) as $[27,28]$

$$
\epsilon=\frac{S_{1}}{\Phi_{1}}+\frac{S_{2}}{\Phi_{2}}
$$

where $S_{l}$ is the spectral efficiency of RX $l$ and $\Phi_{l}$ is the average power to transmit symbol $s_{l}$ to $\mathrm{RX} l$ defined as

$$
\begin{aligned}
\Phi_{l} & =\frac{\left(p_{1}^{\left(t_{l, 1}\right)}+P_{1}^{\left(t_{l, 1}\right)}\right) T+\left(p_{2}^{\left(t_{l, 2}\right)}+P_{2}^{\left(t_{l, 2}\right)}\right) T}{N T} \\
& =\frac{\left(p_{1}^{\left(t_{l, 1}\right)}+P_{1}^{\left(t_{l, 1}\right)}\right)+\left(p_{2}^{\left(t_{l, 2}\right)}+P_{2}^{\left(t_{l, 2}\right)}\right)}{N}
\end{aligned}
$$

where $T$ is the time slot duration, $N$ is the total number of time slots per transmission round, and $t_{l, m}$ is the time slot in which symbol $s_{l}$ is transmitted by TX $m{ }^{1}$ To avoid a cumbersome notation, we will simply use $t$ without a subscript. With Eqs. (1) and (2), we can establish a fair comparison among schemes that use a different number of time slots per transmission round.

Our objective is then to maximize $\epsilon$ subject to transmit power and minimum spectral efficiency constraints:

$$
\begin{aligned}
& \underset{P_{1}^{(t)}, P_{2}^{(t)} \forall t}{\operatorname{maximize}} \epsilon \\
& \text { s.t. } 0 \leq P_{1}^{(t)} \leq P_{1}^{\max }, \forall t, \\
& 0 \leq P_{2}^{(t)} \leq P_{2}^{\max }, \forall t, \\
& S_{1} \geq S_{1}^{\min }, \\
& S_{2} \geq S_{2}^{\min } .
\end{aligned}
$$

\section{Baseline approaches that do not exploit the overhearing capabilities of TXs}

This section presents the analysis of the baseline approaches, which will be used in Section 6 to compare the performance of the overhearing approaches presented in Section 4.

\subsection{TDMA}

In a basic time division multiple access (TDMA) approach, the communication is done in turns, i.e., first TX 1 transmits $s_{1}$ to RX 1 while TX 2 is inactive, and then TX 2 transmits $s_{2}$ to RX 2 while TX 1 is inactive, hence requiring two time slots per transmission round.

The energy efficiency maximization of TDMA is given as

$$
\begin{aligned}
\underset{P_{1}^{(1)}, P_{2}^{(2)}}{\operatorname{maxime}} \epsilon^{\mathrm{TDMA}} & =\frac{S_{1}^{\mathrm{TDMA}}}{\frac{1}{2}\left(p_{1}^{(1)}+P_{1}^{(1)}\right)}+\frac{S_{2}^{\mathrm{TDMA}}}{\frac{1}{2}\left(p_{2}^{(2)}+P_{2}^{(2)}\right)} \\
\text { s.t. } 0 \leq P_{l}^{(l)} & \leq P_{l}^{\max } \\
S_{l}^{\mathrm{TDMA}} & \geq S_{l}^{\min }
\end{aligned}
$$

for $l=\{1,2\}$ where

$$
S_{l}^{\mathrm{TDMA}}=\frac{1}{2} \log _{2}\left(1+\gamma_{l l}^{(l)}\right)
$$

and the $\frac{1}{2}$ pre-log factor comes from the two time slots required. Since the two transmissions are fully decoupled, the energy efficiency is maximized independently for each term of Eq. (4).

In the transmit power-dominated regime, $P_{1}^{(1)} \gg p_{1}^{(1)}$ and $P_{2}^{(2)} \gg p_{2}^{(2)}$ and we can see that Eq. (4) is strictly decreasing with both $P_{1}^{(1)}$ and $P_{2}^{(2)}$ (see Appendix A with $a=\left|h_{l l}\right|^{2}$ and $k=b=0$ ). In this case, the maximization of the energy efficiency corresponds to using the following transmit powers to satisfy the minimum spectral efficiency constraints

$$
P_{l}^{(l)}= \begin{cases}\frac{2^{2 S_{l}^{\min }}-1}{\left|h_{l l}\right|^{2}}, & \text { if } \frac{2^{2 S_{l}^{\min }}-1}{\left|h_{l l}\right|^{2}} \leq P_{l}^{\max } \\ \text { no solution (no success), } & \text { otherwise }\end{cases}
$$

Note the exponent 2 corresponding to the two time slots required per transmission round. This factor 
reveals a penalty in the power of schemes that use several time slots.

In the circuit power-dominated regime, $p_{1}^{(1)} \gg P_{1}^{(1)}$ and $p_{2}^{(2)} \gg P_{2}^{(2)}$, and assuming for simplicity $p_{1}^{(1)}=p_{2}^{(2)}=p^{(t)}$, the energy efficiency maximization of TDMA is given as

$$
\begin{aligned}
\underset{P_{1}^{(1)}, P_{2}^{(2)}}{\operatorname{maximize}} \epsilon^{\mathrm{TDMA}} & \approx \frac{S_{1}^{\mathrm{TDMA}}+S_{2}^{\mathrm{TDMA}}}{\frac{1}{2} p^{(t)}} \\
\text { s.t. } 0 \leq P_{l}^{(l)} & \leq P_{l}^{\max } \\
S_{l}^{\mathrm{TDMA}} & \geq S_{l}^{\min }
\end{aligned}
$$

where the energy efficiency is maximized independently for each term. In this case, the maximization of the energy efficiency corresponds to both TXs transmitting with maximum power, i.e., $P_{1}^{(1)}=P_{1}^{\max }$ and $P_{2}^{(2)}=$ $P_{2}^{\max }$, assuming that the minimum spectral efficiency constraints are satisfied (success rate); otherwise, there is no solution.

\subsection{INTF}

This approach consists in both TXs transmitting simultaneously regardless of the resulting interference, hence requiring one time slot per transmission round. We refer to this approach as INTF (interference).

The energy efficiency maximization of INTF is given as

$$
\begin{aligned}
\underset{P_{1}^{(1)}, P_{2}^{(1)}}{\operatorname{maximize}} \epsilon^{\mathrm{INTF}} & =\frac{S_{1}^{\mathrm{INTF}}}{p_{1}^{(1)}+P_{1}^{(1)}}+\frac{S_{2}^{\mathrm{INTF}}}{p_{2}^{(1)}+P_{2}^{(1)}} \\
\text { s.t. } 0 \leq P_{l}^{(1)} & \leq P_{l}^{\max } \\
S_{l}^{\mathrm{INTF}} & \geq S_{l}^{\min }
\end{aligned}
$$

where

$$
S_{l}^{\mathrm{INTF}}=\log _{2}\left(1+\frac{\gamma_{l l}^{(1)}}{1+\gamma_{m l}^{(1)}}\right)
$$

for $l=\{1,2\}, m=\{2,1\}$, and $l \neq m$.

From the analysis presented in Appendix A (with $a=$ $\left.\frac{\left|h_{l l}\right|^{2}}{1+\gamma_{m l}^{(1)}}, k=b=0\right)$, in the transmit power-dominated regime, i.e., $P_{1}^{(1)} \gg p_{1}^{(1)}$ and $P_{2}^{(1)} \gg p_{2}^{(1)}$, Eq. (8) is strictly decreasing with both $P_{1}^{(1)}$ and $P_{2}^{(1)}$. In this case, the maximization of the energy efficiency corresponds to using the following transmit powers for $l=\{1,2\}, m=\{2,1\}$, and $l \neq m$, where

$$
\Delta_{\mathrm{INTF}}=\frac{\left(2^{S_{l}^{\min }}-1\right)\left(\left|h_{m m}\right|^{2}+\left|h_{l m}\right|^{2}\left(2^{S_{m}^{\min }}-1\right)\right)}{\left|h_{l l}\right|^{2}\left|h_{m m}\right|^{2}-\left|h_{l m}\right|^{2}\left|h_{m l}\right|^{2}\left(2^{S_{1}^{\min }}-1\right)\left(2^{S_{2}^{\min }}-1\right)} .
$$

In the circuit power-dominated regime, $p_{1}^{(1)} \gg P_{1}^{(1)}$ and $p_{2}^{(1)} \gg P_{2}^{(1)}$, and assuming $p_{1}^{(1)}=p_{2}^{(1)}=p^{(1)}$, Eq. (8) can be expressed as

$$
\begin{aligned}
\underset{P_{1}^{(1)}, P_{2}^{(1)}}{\operatorname{maximize}} \epsilon^{\mathrm{INTF}} & =\frac{S_{1}^{\mathrm{INTF}}+S_{2}^{\mathrm{INTF}}}{p^{(1)}} \\
\text { s.t. } 0 \leq P_{l}^{(1)} & \leq P_{l}^{\max } \\
S_{l}^{\mathrm{INTF}} & \geq S_{l}^{\min }
\end{aligned}
$$

which is equivalent to maximizing the sum spectral efficiency in an interference channel. The optimal solution is then binary, i.e., both TXs transmit with maximum power or only one of them with maximum power and the other with zero power depending on the SNR [29].

\section{Approaches that exploit the overhearing capabilities of TXs}

In this section, we analyze the energy efficiency of two approaches (based on [22] and [25]) that exploit the overhearing capabilities of TXs. We refer to them as DIV and DRCP.

\subsection{DIV}

One way of exploiting the overhearing capabilities of the TXs consists in sharing the transmitted symbols between TXs in order to have each symbol retransmitted by a different TX. This approach resembles what was proposed in [22] but using the TXs as relays. For instance, TX 1 transmits $s_{1}$, which is received by RX 1 and TX 2 in time slot 1 , and TX 2 transmits $s_{2}$, which is received by RX 2 and TX 1 in time slot 2 . Then, in time slot 3, TX 1 transmits $s_{2}$, and in time slot 4, TX 2 transmits $s_{1}$. We refer to this approach as DIV (diversity).

Assuming a channel coherence time larger than four time slots, the received signals can be expressed as

$$
\begin{aligned}
& y_{1}^{(1)}=\sqrt{P_{1}^{(1)}} h_{11} s_{1}+n_{1}^{(1)} \\
& y_{2}^{(2)}=\sqrt{P_{2}^{(2)}} h_{21} s_{2}+n_{1}^{(2)} \\
& y_{2}^{(3)}=\sqrt{P_{1}^{(3)}} h_{11} z_{12}+n_{1}^{(3)} \\
& y_{1}^{(4)}=\sqrt{P_{2}^{(4)}} h_{21} z_{21}+n_{1}^{(4)},
\end{aligned}
$$

$$
P_{l}^{(1)}=\left\{\begin{array} { l } 
{ \Delta _ { \mathrm { INTF } } , } \\
{ \text { no solution (no success), } }
\end{array} \quad \text { otherwise } \left\{\begin{array}{l}
\Delta_{\mathrm{INTF}} \leq P_{l}^{\max } \\
\left|h_{l l}\right|^{2}\left|h_{m m}\right|^{2}>\left|h_{l m}\right|^{2}\left|h_{m l}\right|^{2}\left(2^{S_{1}^{\min }}-1\right)\left(2^{S_{2}^{\min }}-1\right)
\end{array}\right.\right.
$$


where $y_{l}^{(t)}$ is the signal received by $\mathrm{RX} l$ in time slot $t, n_{l}^{(t)}$ is the noise received by $\mathrm{RX} l$ in time slot $t$ and

$$
z_{l m}=s_{m}+\frac{n_{l^{\prime}}^{(m)}}{\sqrt{P_{m}^{(m)} g_{m l}}},
$$

where $n_{l^{\prime}}^{(t)}$ is the received noise for TX $l$ in time slot $t$.

The signal received by RX $1, \mathbf{y}_{1}=\mathbf{a}_{1} s_{1}+\mathbf{n}_{1}$, can be expressed in matrix form as

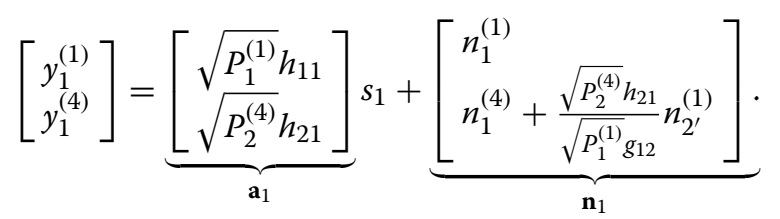

A similar expression can be derived for RX 2 .

The spectral efficiency of DIV using an MMSE estimator (see Section 4.2) is given as

$$
S_{l}^{\text {DIV }}=\frac{1}{4} \log _{2}\left(1+\gamma_{l l}^{\left(t_{1}\right)}+\frac{\gamma_{m l}^{\left(t_{2}\right)}}{\frac{\gamma_{m l}^{\left(t_{2}\right)}}{\xi_{l m}^{\left(t_{1}\right)}}+1}\right)
$$

for $l=\{1,2\}, m=\{2,1\}$, and $l \neq m$ and where $t_{1}=1$ and $t_{2}=4$ for $l=1$ and $t_{1}=2$ and $t_{2}=3$ for $l=2$. Notice that the pre-log factor of $\frac{1}{4}$ is due to the use of four time slots for transmitting both symbols by both TXs but that there is the corresponding SNR gain inside the logarithm.

The energy efficiency maximization of DIV is given as

$$
\begin{aligned}
\underset{P_{1}^{(t)}, P_{2}^{(t)}}{\operatorname{maximize}} \epsilon^{\mathrm{DIV}}= & \frac{S_{1}^{\mathrm{DIV}}}{\frac{1}{4}\left(p_{1}^{(1)}+p_{2}^{(4)}+P_{1}^{(1)}+P_{2}^{(4)}\right)} \\
& +\frac{S_{2}^{\mathrm{DIV}}}{\frac{1}{4}\left(p_{2}^{(2)}+p_{1}^{(3)}+P_{2}^{(2)}+P_{1}^{(3)}\right)} \\
\text { s.t. } 0 \leq P_{l}^{(t)} \leq & P_{l}^{\max } \\
S_{l}^{\mathrm{DIV}} & S_{l}^{\min }
\end{aligned}
$$

In the transmit power-dominated regime, $P_{1}^{(1)}+P_{2}^{(4)} \gg$ $p_{1}^{(1)}+p_{2}^{(4)}$ and $P_{2}^{(2)}+P_{1}^{(3)} \gg p_{2}^{(2)}+p_{1}^{(3)}$, and assuming a strong overhearing link $\xi_{l m}^{(t)} \gg \gamma_{m l}^{(t)}$, we can observe that Eq. (17) has a maximum found by solving for $l=\{1,2\}, m=\{2,1\}$, and $l \neq m$, and where $W(x)$ is the Lambert function of $x$.

For a proof, see Appendix A with $a=\left|h_{l l}\right|^{2}, b=\gamma_{m l}^{(t)}$, and $k=P_{m}^{(t)}$. Note that since Eq. (18) depends on $P_{l}^{(t)}$ and given the exponential growth with respect to $P_{l}^{(t)}$ and $P_{m}^{(t)}$, both $P_{l}^{(t)}$ and $P_{m}^{(t)}$ will tend to be as small as possible. Hence, an approximation consists in selecting whether we use the transmission or the relaying phase to transmit a given symbol with the transmit power that satisfies the spectral efficiency constraints. Therefore, we propose to use the following transmit powers that satisfy the minimum spectral efficiency constraints.

$$
P_{1}^{(1)}= \begin{cases}\frac{2^{4 S_{1}^{\min }}-1}{\left|h_{11}\right|^{2}}, & \text { if }\left\{\begin{array}{l}
\left|h_{11}\right|^{2} \geq\left|h_{21}\right|^{2} \\
\frac{2^{4 S_{1}^{\min }}-1}{\left|h_{11}\right|^{2}} \leq P_{1}^{\max }
\end{array}\right. \\
\frac{2_{1}^{S^{\min }}-1}{\left|g_{12}\right|^{2}}, & \text { if }\left\{\begin{array}{l}
\left|h_{21}\right|^{41_{1}^{\min }}>\left|h_{11}\right|^{2} \\
\frac{2^{4 S_{1}}-1}{\left|h_{21}\right|^{2}} \leq P_{2}^{\max }
\end{array}\right. \\
\text { no solution (no success), } & \text { otherwise }\end{cases}
$$$$
P_{2}^{(2)}= \begin{cases}\frac{2^{4 S_{2}^{\min }}-1}{\left|h_{22}\right|^{2}}, & \text { if }\left\{\begin{array}{l}
\left|h_{22}\right|^{2} \geq\left|h_{12}\right|^{2} \\
\frac{2^{4 S_{2}^{\min }}-1}{\left|h_{22}\right|^{2}} \leq P_{2}^{\max }
\end{array}\right. \\
\frac{2_{2}^{S_{2}^{\min }-1}}{\left|g_{21}\right|^{2}}, & \text { if }\left\{\begin{array}{l}
\left|h_{12}\right|^{2}>\left|h_{22}\right|^{2} \\
\frac{2^{4 S_{2}^{\min }}-1}{\left|h_{12}\right|^{2}} \leq P_{1}^{\max }
\end{array}\right. \\
\text { no solution (no success), } & \text { otherwise }\end{cases}
$$$$
P_{1}^{(3)}= \begin{cases}\frac{2^{4 S_{2}^{\min }-1}}{\left|h_{12}\right|^{2}}, & \text { if }\left\{\begin{array}{l}
\left|h_{12}\right|^{2}>\left|h_{22}\right|^{2} \\
\frac{2^{4 S_{2}^{\min }}-1}{\left|h_{12}\right|^{2}} \leq P_{1}^{\max }
\end{array}\right. \\
0, & \text { if }\left\{\begin{array}{l}
\left|h_{22}\right|^{2} \geq\left|h_{12}\right|^{2} \\
\frac{2_{2}^{\min }-1}{\left|h_{22}\right|^{2}} \leq P_{2}^{\max }
\end{array}\right. \\
\text { no solution (no success), } & \text { otherwise }\end{cases}
$$

$$
P_{2}^{(4)}= \begin{cases}\frac{2^{4 S_{1}^{\min }}-1}{\left|h_{21}\right|^{2}}, & \text { if }\left\{\begin{array}{l}
\left|h_{21}\right|^{2}>\left|h_{11}\right|^{2} \\
\frac{2^{4 s_{1}^{\min }}-1}{\left|h_{21}\right|^{2}} \leq P_{2}^{\max }
\end{array}\right. \\
0, & \text { if }\left\{\begin{array}{l}
\left|h_{11}\right|^{4} \geq\left|h_{21}\right|^{2} \\
\frac{2^{4 S_{1}^{\min }}-1}{\left|h_{11}\right|^{2}} \leq P_{1}^{\max }
\end{array}\right. \\
\text { no solution (no success), } & \text { otherwise }\end{cases}
$$

Note that the minimum value in the first two time slots needs to be sufficiently large to be decoded by the overhearing TX and the exponent 4 corresponding to the four time slots required per transmission round. We will later compare this approximation with the optimal solution

$$
P_{l}^{(t)}= \begin{cases}\frac{e^{W\left(\left|h_{l l}\right|^{2}\left(P_{m}^{(t)}+P_{l}^{(t)}\right)\right)_{1}-1-P_{m}^{(t)}\left|h_{m l}\right|^{2}},}{\left|h_{l l}\right|^{2}}, & \begin{array}{l}
\frac{e^{W\left(\left|h_{l l}\right|^{2}\left(P_{m}^{(t)}+P_{l}^{(t)}\right)\right)}-1-P_{m}^{(t)}\left|h_{m l}\right|^{2}}{\left|h_{l l}\right|^{2}} \leq P_{l}^{\max } \\
e^{W\left(\left|h_{l l}\right|^{2}\left(P_{m}^{(t)}+P_{l}^{(t)}\right)\right)} \geq 1+P_{m}^{(t)}\left|h_{m l}\right|^{2} \\
P_{l}^{(t)}\left|h_{l l}\right|^{2}+P_{m}^{(t)}\left|h_{m l}\right|^{2} \geq 2^{4 S_{l}^{\min }}-1
\end{array} \\
\text { no solution (no success), } & \text { otherwise, }\end{cases}
$$


obtained through an exhaustive search over all possible transmit power combinations.

In the circuit power-dominated regime, $P_{1}^{(1)}+P_{2}^{(4)} \ll$ $2 p^{(t)}$ and $P_{2}^{(2)}+P_{1}^{(3)} \ll 2 p^{(t)}$, and assuming $p_{1}^{(t)}=p_{2}^{(t)}=$ $p^{(t)}$, Eq. (17) can be expressed as

$$
\begin{aligned}
\underset{P_{1}^{(t)}, P_{2}^{(t)}}{\operatorname{maximize}} \epsilon^{\mathrm{DIV}} & =\frac{S_{1}^{\mathrm{DIV}}+S_{2}^{\mathrm{DIV}}}{\frac{1}{4}\left(2 p^{(t)}\right)} \\
\text { s.t. } 0 \leq P_{l}^{(t)} & \leq P_{l}^{\max } \\
S_{l}^{\mathrm{DIV}} & \geq S_{l}^{\min }
\end{aligned}
$$

where the energy efficiency is maximized independently for each term, which corresponds to both TXs transmitting with maximum power, i.e., $P_{1}^{(1)}=P_{1}^{(3)}=P_{1}^{\max }$ and $P_{2}^{(2)}=P_{2}^{(4)}=P_{2}^{\max }$. If the spectral efficiency constraints are not satisfied, we assume that there is no solution.

\subsection{DRCP}

A different way of exploiting the overhearing capabilities of the TXs without requiring so many time slots as DIV is proposed in [25]. The DRCP is shown to increase the spectral efficiency by finding a balance between spatial diversity and transmission time. During the transmission phase, each TX transmits a symbol in a dedicated time slot. Then, during the relaying phase, all the TXs transmit simultaneously the overheard symbol. Specifically, in time slot 1 , TX 1 transmits $s_{1}$ to both RXs and to TX 2. In time slot 2, TX 2 transmits $s_{2}$ to both RXs and to TX 1 . In time slot 3 , each TX acts as a relay to transmit the overheard symbol ( $s_{2}$ for TX 1 and $s_{1}$ for TX 2) to both RXs.

Assuming a channel coherence time larger than three time slots, the received signals for RX 1 in the three time slots can be expressed as

$$
\begin{aligned}
& y_{1}^{(1)}=\sqrt{P_{1}^{(1)}} h_{11} s_{1}+n_{1}^{(1)} \\
& y_{1}^{(2)}=\sqrt{P_{2}^{(2)}} h_{21} s_{2}+n_{1}^{(2)} \\
& y_{1}^{(3)}=\sqrt{P_{1}^{(3)}} h_{11} z_{12}+\sqrt{P_{2}^{(3)}} h_{21} z_{21}+n_{1}^{(3)} .
\end{aligned}
$$

The signal received by RX $1, \mathbf{y}_{1}=\mathbf{a}_{1} s_{1}+\mathbf{a}_{2} s_{2}+\mathbf{n}_{1}=$ $\mathbf{a}_{1} s_{1}+\mathbf{w}_{1}$, can be expressed in matrix form as

$$
\begin{aligned}
& {\left[\begin{array}{l}
y_{1}^{(1)} \\
y_{1}^{(2)} \\
y_{1}^{(3)}
\end{array}\right]=\left[\begin{array}{ll}
\sqrt{P_{1}^{(1)}} h_{11} & 0 \\
0 & \sqrt{P_{2}^{(2)}} h_{21} \\
\sqrt{P_{2}^{(3)}} h_{21} & \underbrace{\sqrt{P_{1}^{(3)}} h_{11}}_{\mathbf{a}_{1}}
\end{array}\right]\left[\begin{array}{l}
s_{1} \\
s_{2}
\end{array}\right]} \\
& +\underbrace{\left[\begin{array}{l}
n_{1}^{(1)} \\
n_{1}^{(2)} \\
n_{1}^{(3)}+\frac{\sqrt{P_{1}^{(3)}} h_{11}}{\sqrt{P_{2}^{(2)}} g_{21}} n_{1^{\prime}}^{(2)}+\frac{\sqrt{P_{2}^{(3)}} h_{21}}{\sqrt{P_{1}^{(1)}} g_{12}} n_{2^{\prime}}^{(1)}
\end{array}\right]}_{\mathbf{n}_{1}} .
\end{aligned}
$$

where $\mathbf{w}_{1}$ is defined as the interference-plus-noise vector of RX 1. A similar expression can be derived for RX 2 .

The MMSE is well known for being an efficient lowcomplexity linear receiver [30]. The MMSE of RX 1 can be derived in two steps. The first step consists in whitening the colored noise term $\mathbf{w}_{1}$ with its covariance matrix $\mathbf{R}_{w_{1}}$. This is achieved by filtering the received signal $\mathbf{y}_{1}$ with the matrix $\mathbf{R}_{w_{1}}^{-1 / 2}$ and then performing maximal ratio combining (MRC) over the remaining signal by taking the inner product of the resulting signal and the vector $\mathbf{R}_{w_{1}}^{-1 / 2} \mathbf{a}_{1}$, resulting in

$$
\begin{aligned}
\hat{z}=\left(\mathbf{R}_{w_{1}}^{-1 / 2} \mathbf{a}_{1}\right)^{H} \mathbf{R}_{w_{1}}^{-1 / 2} \mathbf{y}_{1} & =\mathbf{a}_{1}^{H} \mathbf{R}_{w_{1}}^{-1} \mathbf{a}_{1} s_{1}+\mathbf{a}_{1}^{H} \mathbf{R}_{w_{1}}^{-1} \mathbf{w}_{1} \\
& =\hat{z}_{\text {sig }}+\hat{z}_{\text {noise }}
\end{aligned}
$$

The signal-to-noise ratio (SNR) of RX 1 using the MMSE is found to be

$$
\mathrm{SNR}_{1}^{\mathrm{DRCP}}=\frac{\mathbb{E}\left\{\left|\hat{z}_{\text {sig }}\right|^{2}\right\}}{\mathbb{E}\left\{\left|\hat{z}_{\text {noise }}\right|^{2}\right\}}=\mathbf{a}_{1}^{H} \mathbf{R}_{w_{1}}^{-1} \mathbf{a}_{1} \sigma_{\mathrm{s}}^{2},
$$

which, as shown in Appendix B, results in

$$
\mathrm{SNR}_{l}^{\mathrm{DRCP}}=\gamma_{l l}^{(l)}+\frac{\gamma_{m l}^{(3)}}{\frac{\gamma_{l l}^{(3)}}{\gamma_{m l}^{(m)}+1}+\frac{\gamma_{l}^{(3)}}{\xi_{m l}^{(m)}}+\frac{\gamma_{m l}^{(3)}}{\xi_{l m}^{(l)}}+1} .
$$

for $l=\{1,2\}, m=\{2,1\}$, and $l \neq m$.

The energy efficiency maximization of DRCP is given as

$$
\begin{aligned}
\underset{P_{1}^{(t)}, P_{2}^{(t)}}{\operatorname{maximize}} \epsilon^{\mathrm{DRCP}}= & \frac{S_{1}^{\mathrm{DRCP}}}{\frac{1}{3}\left(p_{1}^{(1)}+p_{2}^{(3)}+P_{1}^{(1)}+P_{2}^{(3)}\right)} \\
& +\frac{S_{2}^{\mathrm{DRCP}}}{\frac{1}{3}\left(p_{2}^{(2)}+p_{1}^{(3)}+P_{2}^{(2)}+P_{1}^{(3)}\right)}
\end{aligned}
$$

$$
\begin{aligned}
\text { s.t. } 0 \leq P_{l}^{(t)} & \leq P_{l}^{\max } \\
S_{l}^{\mathrm{DRCP}} & \geq S_{l}^{\min }
\end{aligned}
$$

where

$$
S_{l}^{\mathrm{DRCP}}=\frac{1}{3} \log _{2}\left(1+\mathrm{SNR}_{l}^{\mathrm{DRCP}}\right) .
$$

Finding the globally optimal solution of (26) requires the joint optimization over different time slots and TXs. For this purpose, we analyze the energy efficiency of DRCP for different cases and propose an approximate solution for each of them. In Section 6, we then compare our approximation with the global optimum that is found by an exhaustive search over all possible power combinations. In all cases, we assume a stonger link between TXs than between TXs and RXs, i.e., $\xi_{n p}^{(t)} \gg \gamma_{l m}^{(t)} \forall l, m, n, p, t$.

\subsubsection{Transmit power-dominated regime}

Case with dominant direct links. When the direct links are stronger than the indirect links, i.e., $\left|h_{11}\right|^{2}>\left|h_{21}\right|^{2}$ 
and $\left|h_{22}\right|^{2}>\left|h_{12}\right|^{2}$, we can approximate the spectral efficiency (27) as

$$
S_{l}^{\mathrm{DRCP}} \approx \frac{1}{3} \log _{2}\left(1+\gamma_{l l}^{(l)}\right)
$$

With this approximation, $P_{1}^{(3)}$ and $P_{2}^{(3)}$ only decrease the energy efficiency of the system, so we set them as $P_{1}^{(3)}=0$ and $P_{2}^{(3)}=0$. Note that the minimum spectral efficiency constraints will be satisfied with the transmission of the first two time slots, however introducing a penalty by leaving the last time slot unused.

Similar to the TDMA case, (26) is strictly decreasing with both $P_{1}^{(1)}$ and $P_{2}^{(2)}$ (for a proof, see Appendix A and use $a=\left|h_{l l}\right|^{2}$ and $k=b=0$ ). In this case, the energy efficiency maximization corresponds to using the following transmit power that satisfy the minimum spectral efficiency constraints for $l=\{1,2\}$ and $t=\{1,2\}$.

$$
P_{l}^{(t)}= \begin{cases}\frac{2^{3 S_{l}^{\min }}-1}{\left|h_{l l}\right|^{2}}, & \text { if } \frac{2^{3 S_{l}^{\min }}-1}{\left|h_{l l}\right|^{2}} \leq P_{l}^{\max } \\ \text { no solution (no success), } & \text { otherwise }\end{cases}
$$

Notice the exponent 3 corresponding to the three time slots required per transmission round.

Case with one dominant direct link and one dominant indirect link. If for one RX the direct link is stronger than the indirect link whereas for the other RX the indirect link is stronger than the direct link, specifically either (i) $\left|h_{11}\right|^{2}>\left|h_{21}\right|^{2}$ and $\left|h_{12}\right|^{2}>\left|h_{22}\right|^{2}$ or (ii) $\left|h_{22}\right|^{2}>\left|h_{12}\right|^{2}$ and $\left|h_{21}\right|^{2}>\left|h_{11}\right|^{2}$, we can approximate the spectral efficiency (27) as

$$
\begin{aligned}
& S_{1}^{\mathrm{DRCP}} \approx \frac{1}{3} \log _{2}\left(1+\gamma_{11}^{(1)}\right) \\
& S_{2}^{\mathrm{DRCP}} \approx \frac{1}{3} \log _{2}\left(1+\gamma_{12}^{(3)}\right)
\end{aligned}
$$

for case (i). For TX 2, it is clear that its transmission only decreases the energy efficiency of the system. For TX 1 , (26) is strictly decreasing with $P_{1}^{(1)}$ and $P_{1}^{(3)}$. In this case, we can set the transmit powers as follows:

$$
\begin{aligned}
P_{1}^{(1)} & = \begin{cases}\frac{2^{3 S_{1}^{\min }}-1}{\left|h_{11}\right|^{2}}, & \text { if } \frac{2^{3 S_{1}^{\min }}-1}{\left|h_{11}\right|^{2}} \leq P_{1}^{\max } \\
\text { no solution (no success) } & \text { otherwise }\end{cases} \\
P_{2}^{(2)} & = \begin{cases}\frac{2^{S_{2}^{\min }}-1}{\left|g_{21}\right|^{2}}, & \text { if } \frac{2^{3 S_{2}^{\min }}-1}{\left|h_{12}\right|^{2}} \leq P_{1}^{\max } \\
\text { no solution (no success) } & \text { otherwise }\end{cases} \\
P_{1}^{(3)} & = \begin{cases}\frac{2^{3 S_{2}^{\min }}-1}{\left|h_{12}\right|^{2}}, & \text { if } \frac{2^{3 S_{2}^{\min }}-1}{\left|h_{12}\right|^{2}} \leq P_{1}^{\max } \\
\text { no solution (no success) } & \text { otherwise }\end{cases} \\
P_{2}^{(3)} & = \begin{cases}0, & \text { if } \frac{2^{3 S_{1}^{\min }}-1}{\left|h_{11}\right|^{2}} \leq P_{1}^{\max } \\
\text { no solution (no success) } & \text { otherwise }\end{cases}
\end{aligned}
$$

Note that in this case, $P_{2}^{(2)}$ needs to be sufficiently large to be decoded by the other TX. For case (ii), we can set the transmit powers as follows:

$$
\begin{aligned}
& P_{1}^{(1)}= \begin{cases}\frac{2_{1}^{S_{1}^{\min }}-1}{\left|g_{12}\right|^{2}}, & \text { if } \frac{2^{3 S_{1}^{\min }}-1}{\left|h_{21}\right|^{2}} \leq P_{2}^{\max } \\
\text { no solution (no success) } & \text { otherwise }\end{cases} \\
& P_{2}^{(2)}= \begin{cases}\frac{2^{3 S_{2}^{\min }}-1}{\left|h_{22}\right|^{2}}, & \text { if } \frac{2^{3 S_{2}^{\min }}-1}{\left|h_{22}\right|^{2}} \leq P_{2}^{\max } \\
\text { no solution (no success) } & \text { otherwise }\end{cases} \\
& P_{1}^{(3)}= \begin{cases}0, & \text { if } \frac{2^{3 S_{2}^{\min }}-1}{\left|h_{22}\right|^{2}} \leq P_{2}^{\max } \\
\text { no solution (no success) } & \text { otherwise }\end{cases} \\
& P_{2}^{(3)}= \begin{cases}\frac{2^{3 S_{1}^{\min }}-1}{\left|h_{21}\right|^{2}}, & \text { if } \frac{2^{3 S_{1}^{\min }}-1}{\left|h_{21}\right|^{2}} \leq P_{2}^{\max } \\
\text { no solution (no success) } & \text { otherwise }\end{cases}
\end{aligned}
$$

Note that in this case, $P_{1}^{(1)}$ needs to be sufficiently large to be decoded by the other TX.

Case with dominant indirect links. In this case, the indirect links are stronger than the direct links, i.e., $\left|h_{21}\right|^{2}>\left|h_{11}\right|^{2}$ and $\left|h_{12}\right|^{2}>\left|h_{22}\right|^{2}$. This case occurs, for instance, when users from a HetNet are closer to an interfering base station than to their serving base station ${ }^{2}$. Different from the previous cases where all the symbols are transmitted in dedicated time slots, here, simultaneous transmissions through the indirect channels occur in the third time slot, generating interference to both RXs. Therefore, we consider two subcases. If one indirect link is stronger than the other by a factor $\beta>1$, i.e., $\left|h_{l m}\right|^{2}>$ $\beta\left|h_{m l}\right|^{2}$, we will allow only TX $l$ to use the third time slot. Otherwise, both TXs use the third time slot.

For the first subcase, when $\left|h_{12}\right|^{2}>\beta\left|h_{21}\right|^{2}$, we can approximate the spectral efficiency (27) as Eq. (30). Therefore, we can use the transmit powers of Eq. (31). Similarly, when $\left|h_{21}\right|^{2}>\beta\left|h_{12}\right|^{2}$, we will use the transmit powers of Eq. (32).

For the second subcase, we can approximate the spectral efficiency (27) as

$$
S_{l}^{\mathrm{DRCP}} \approx \frac{1}{3} \log _{2}\left(1+\gamma_{m l}^{(3)}\right)
$$

for $l=\{1,2\}, m=\{2,1\}$, and $l \neq m$. In this case, we will use the third time slot for simultaneous transmissions. Since in this case, $P_{1}^{(1)}$ and $P_{2}^{(2)}$ only decrease the energy efficiency of the system, we need to make sure that they are sufficiently large to be decoded by the other TX: 


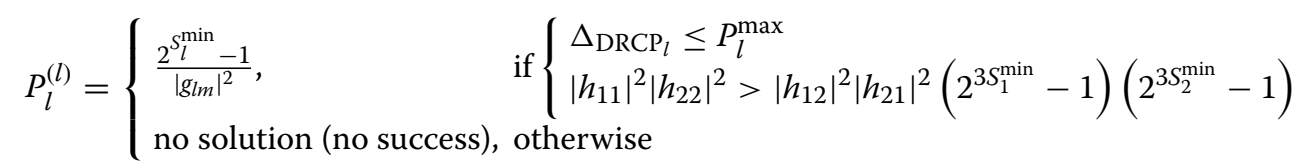

where

$$
\Delta_{\mathrm{DRCP}_{l}}=\frac{\left(2^{3 S_{l}^{\min }}-1\right)\left(\left|h_{m m}\right|^{2}+\left|h_{l m}\right|^{2}\left(2^{3 S_{m}^{\min }}-1\right)\right)}{\left|h_{l l}\right|^{2}\left|h_{m m}\right|^{2}-\left|h_{l m}\right|^{2}\left|h_{m l}\right|^{2}\left(2^{3 S_{1}^{\min }}-1\right)\left(2^{3 S_{2}^{\min }}-1\right)}
$$

for $l=\{1,2\}, m=\{2,1\}$, and $l \neq m$.

Furthermore, since (26) is strictly decreasing with $P_{1}^{(3)}$ and $P_{2}^{(3)}$ (as seen in Appendix A with $a=\left|h_{m l}\right|^{2}$ for $m \neq l$ and $k=b=0$ ), we propose to use the following transmit powers that satisfy the minimum spectral efficiency constraints

$$
\begin{aligned}
& P_{l}^{(3)}= \\
& \left\{\begin{array} { l } 
{ \Delta _ { \mathrm { DRCP } _ { l } , } } \\
{ \text { no solution (no success), otherwise } }
\end{array} \quad \text { if } \left\{\begin{array}{l}
\Delta_{\mathrm{DRCP}_{l} \leq P_{l}^{\max }} \\
\left|h_{l l}\right|^{2}\left|h_{m m}\right|^{2}>\left|h_{l m}\right|^{2}\left|h_{m l}\right|^{2}\left(2^{3 S_{1}^{\min }}-1\right)\left(2^{3 S_{2}^{\min }}-1\right)
\end{array}\right.\right.
\end{aligned}
$$

for $l=\{1,2\}, m=\{2,1\}$, and $l \neq m$. Note that (36) is similar to (10) for INTF except for the penalty of requiring three time slots per transmission round.

\subsubsection{Circuit power-dominated regime}

In the circuit power-dominated regime, we can assume that $p_{1}^{(1)}+p_{2}^{(3)} \gg P_{1}^{(1)}+P_{2}^{(3)}$ and $p_{2}^{(2)}+p_{1}^{(3)} \gg P_{2}^{(2)}+P_{1}^{(3)}$. For simplicity, we assume $p_{1}^{(1)}=p_{2}^{(2)}=p_{1}^{(3)}=p_{2}^{(3)}=p^{(t)}$ and we can see that (26) can be approximated as

$$
\begin{aligned}
\underset{P_{1}^{(t)}, P_{2}^{(t)} \forall t}{\operatorname{maximize}} \epsilon^{\mathrm{DRCP}} & \approx \frac{S_{1}^{\mathrm{DRCP}}+S_{2}^{\mathrm{DRCP}}}{\frac{1}{3}\left(2 p^{(t)}\right)} \\
\text { s.t. } 0 \leq P_{l}^{(t)} & \leq P_{l}^{\max } \\
S_{l}^{\mathrm{DRCP}} & \geq S_{l}^{\min }
\end{aligned}
$$

where the energy efficiency is maximized independently for each term of Eq. (37).

Case with dominant direct links. When the direct links are stronger than the indirect links, i.e., $\left|h_{11}\right|^{2}>\left|h_{21}\right|^{2}$ and $\left|h_{22}\right|^{2}>\left|h_{12}\right|^{2}$, we can approximate the spectral efficiency as Eq. (28).

For the first two time slots, this corresponds to both TXs using maximum transmit power, i.e., $P_{1}^{(1)}=P_{1}^{\max }$ and $P_{2}^{(2)}=P_{2}^{\max }$. For the third time slot, $P_{1}^{(3)}$ and $P_{2}^{(3)}$ only decrease the energy efficiency of the system. Therefore, we propose to set $P_{1}^{(3)}=0$ and $P_{2}^{(3)}=0$. If the spectral efficiency constraints are not satisfied, we assume that there is no solution.

Case with one dominant direct link and one dominant indirect link. This is the case where either (i) $\left|h_{11}\right|^{2}>$ $\left|h_{21}\right|^{2}$ and $\left|h_{12}\right|^{2}>\left|h_{22}\right|^{2}$ or (ii) $\left|h_{22}\right|^{2}>\left|h_{12}\right|^{2}$ and $\left|h_{21}\right|^{2}>\left|h_{11}\right|^{2}$.
For case (i), we can approximate the spectral efficiency as (30). With this approximation, the energy efficiency is maximized independently for each term of (37), which corresponds to $P_{1}^{(1)}=P_{1}^{\max }, P_{1}^{(3)}=P_{1}^{\max }, P_{2}^{(2)}=\frac{2_{2}^{S_{2}^{\min }}}{\left|g_{21}\right|^{2}}$, and $P_{2}^{(3)}=0$. For case (ii), we set $P_{2}^{(2)}=P_{2}^{\max }, P_{2}^{(3)}=P_{2}^{\max }$, $P_{1}^{(1)}=\frac{2^{S_{1}^{\min }}}{\left|g_{12}\right|^{2}}$, and $P_{1}^{(3)}=0$. For both cases, if the spectral efficiency constraints are not satisfied, we assume that there is no solution.

Case with dominant indirect links. This is the case when the indirect links are stronger than the direct links, i.e., $\left|h_{21}\right|^{2}>\left|h_{11}\right|^{2}$ and $\left|h_{12}\right|^{2}>\left|h_{22}\right|^{2}$. We consider again two subcases. When $\left|h_{12}\right|^{2}>\beta\left|h_{21}\right|^{2}$, we can approximate the spectral efficiency as Eq. (30) and we use the following transmit powers:

$$
\begin{aligned}
& P_{1}^{(1)}= \begin{cases}P_{1}^{\max }, & \text { if } \frac{2^{3 S_{1}^{\min }}-1}{\left|h_{11}\right|^{2}} \leq P_{1}^{\max } \\
\text { no solution (no success), } & \text { otherwise }\end{cases} \\
& P_{2}^{(2)}= \begin{cases}\frac{2^{S_{1}^{\min }}-1}{\left|g_{21}\right|^{2}}, & \text { if } \frac{2^{3 S_{2}^{\min }}-1}{\left|h_{12}\right|^{2}} \leq P_{1}^{\max } \\
\text { no solution (no success), } & \text { otherwise }\end{cases} \\
& P_{1}^{(3)}= \begin{cases}P_{1}^{\max }, & \text { if } \frac{2^{3 S_{2}^{\min }}-1}{\left|h_{12}\right|^{2}} \leq P_{1}^{\max } \\
\text { no solution (no success) } & \text { otherwise }\end{cases} \\
& P_{2}^{(3)}= \begin{cases}0, & \text { if } \frac{2^{3 S_{1}^{\min }}-1}{\left|h_{11}\right|^{2}} \leq P_{1}^{\max } \\
\text { no solution (no success), } & \text { otherwise }\end{cases}
\end{aligned}
$$


Similarly, if $\left|h_{21}\right|^{2}>\beta\left|h_{12}\right|^{2}$, we use

$$
\begin{aligned}
& P_{1}^{(1)}= \begin{cases}\frac{2^{S_{2}^{\min }}-1}{\left|g_{12}\right|^{2}}, & \text { if } \frac{2^{3 S_{1}^{\min }}-1}{\left|h_{21}\right|^{2}} \leq P_{2}^{\max } \\
\text { no solution (no success) } & \text { otherwise }\end{cases} \\
& P_{2}^{(2)}= \begin{cases}P_{2}^{\max }, & \text { if } \frac{2^{3 S_{2}^{\min }}-1}{\left|h_{22}\right|^{2}} \leq P_{2}^{\max } \\
\text { no solution (no success), } & \text { otherwise }\end{cases} \\
& P_{1}^{(3)}= \begin{cases}0, & \text { if } \frac{2^{3 S_{2}^{\min }}-1}{\left|h_{22}\right|^{2}} \leq P_{2}^{\max } \\
\text { no solution (no success) } & \text { otherwise }\end{cases} \\
& P_{2}^{(3)}= \begin{cases}P_{2}^{\max }, & \text { if } \frac{2^{3 S_{1}^{\min }}-1}{\left|h_{21}\right|^{2}} \leq P_{2}^{\max } \\
\text { no solution (no success), } & \text { otherwise }\end{cases}
\end{aligned}
$$

In the second subcase, we can approximate the spectral efficiency as Eq. (33), where $P_{1}^{(1)}$ and $P_{2}^{(2)}$ only decrease the energy efficiency of the system. Therefore, we only need to make sure that they are sufficiently large to be decoded by the other TX as in Eq. (34). For the third time slot, given that these are simultaneous transmissions as in INTF, the optimal solution is binary [29]. Again, if the spectral efficiency constraints are not satisfied, we assume that there is no solution.

The whole DRCP power control strategy for the different cases can be summarized in Algorithm 1.

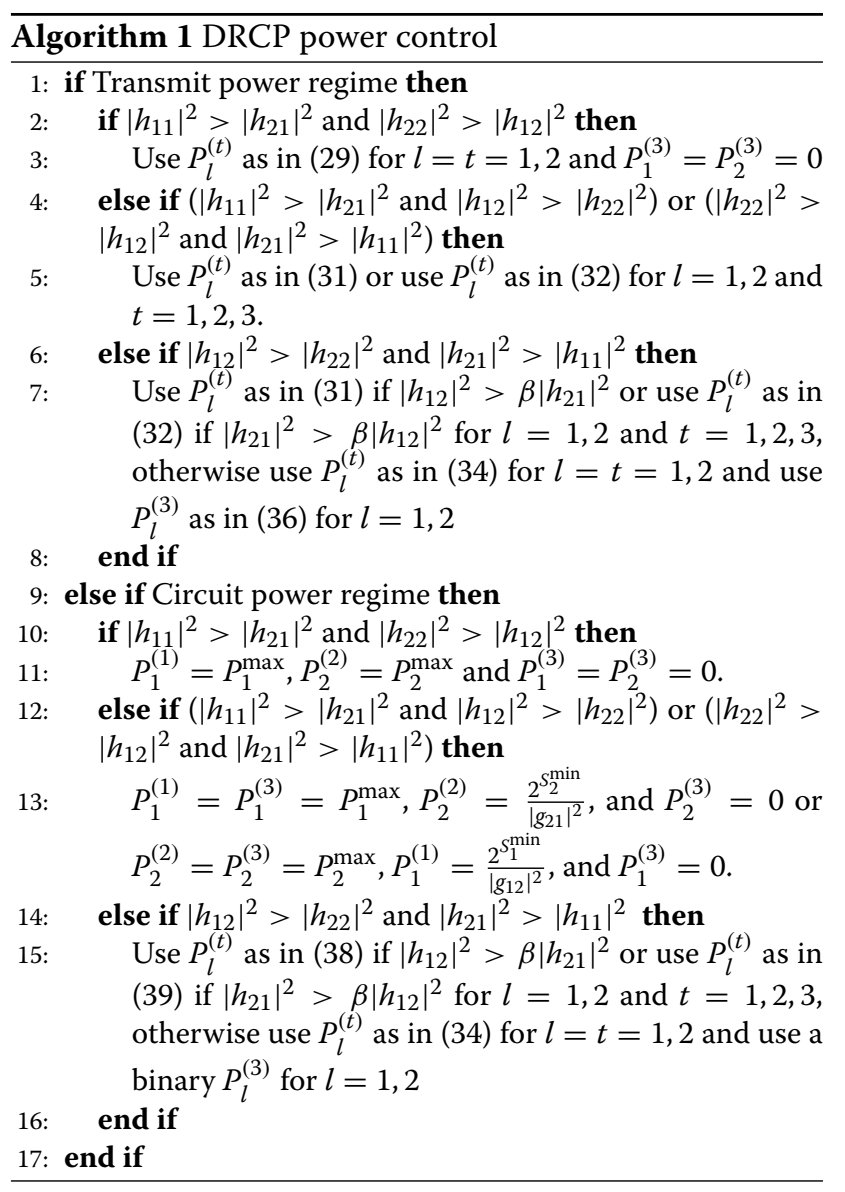

\section{Complexity analysis}

As seen in previous sections, knowing the optimal power control strategy for DIV and DRCP requires an exhaustive search over all the possible power values in every time slot and for every TX. In contrast, with the proposed sub-optimal power control strategy, we divide the problem into two power regimes and classify it according to the dominance of certain links. Based on this, we select the closed-form formula that computes the power for every TX.

For instance, for the system in consideration, with two TXs and two RXs, we are looking for four power values for DIV and also four power values for DRCP. Assuming that each TX can use one of $K$ possible transmit power values, an exhaustive search that can lead to the optimal power control yields up to $K^{4}$ different transmit power combinations for both DIV and DRCP (the higher the $K$, the higher the accuracy).

The proposed sub-optimal power control strategy for both DIV and DRCP classifies the scenario according to the dominance of the transmit power or the circuit power and according to the dominance of certain links (direct or indirect). According to this classification, the proposed power control strategy obtains the corresponding power value for each TX through a closed-form formula. Specifically for DIV, this results in using the formulas of (19) when the system works in the transmit power-dominated regime and using maximum power for each TX when the system works in the circuit power-dominated regime. Specifically for DRCP, the proposed power control strategy obtains the corresponding power value for each TX through Algorithm 1. Hence, instead of performing $K^{4}$ operations to obtain the optimal power control, we only need to classify the scenario (regime and links dominance) and then use a closed-form formula to obtain a suboptimal power control. This complexity reduction comes with a small degradation in spectral efficiency, energy efficiency, and success rate with respect to the optimal power control strategy as can be seen in Section 6.

\section{Performance evaluation}

In this section, we compare the approaches analyzed in previous sections in terms of energy efficiency, spectral efficiency, and success rate (probability of reaching a solution that satisfies the constraints). We consider a Rayleigh fading channel model with $\mathbb{E}\left\{\left|g_{12}\right|^{2}\right\}=\mathbb{E}\left\{\left|g_{21}\right|^{2}\right\}=100$ and direct links with $\mathbb{E}\left\{\left|h_{11}\right|^{2}\right\}=\mathbb{E}\left\{\left|h_{22}\right|^{2}\right\}=1$ (we will only vary the indirect link average gain). We use a fixed circuit power of $p_{1}^{(t)}=p_{2}^{(t)}=0.1 \mathrm{~W} \forall t$ with $\sigma_{\mathrm{s}}^{2}=\sigma_{\mathrm{n}}^{2}=1$, $\beta=3$, and $S_{1}^{\min }=S_{2}^{\min }=0.3$ bits $/ \mathrm{Hz}$.

We consider the two regimes studied in this paper: transmit power-dominated and circuit power-dominated. In the first one, the maximum transmit power of both TXs is $10 \mathrm{~W}$. In the second one, it is $0.05 \mathrm{~W}$. In order to 
evaluate the performance under different conditions, we first vary the average of both indirect links equally, i.e., $R_{1}=\mathbb{E}\left\{\left|h_{21}\right|^{2}\right\}=\mathbb{E}\left\{\left|h_{12}\right|^{2}\right\}$. As a second example, we vary the average of one indirect link relative to the average of the other indirect link, i.e., $R_{2}=\frac{1}{\mathbb{E}\left\{\left|h_{21}\right|^{2}\right\}}=\mathbb{E}\left\{\left|h_{12}\right|^{2}\right\}$. For the circuit power-dominated regime, we vary only $R_{1}$ (with a gain 10 times larger for all the links). In the case of DIV and DRCP, we include the optimal solution obtained with an exhaustive search over all the possible power combinations, named respectively "DIV optimal" and "DRCP optimal." Finally, considering all equal average links, i.e., $\mathbb{E}\left\{\left|h_{11}\right|^{2}\right\}=\mathbb{E}\left\{\left|h_{12}\right|^{2}\right\}=\mathbb{E}\left\{\left|h_{21}\right|^{2}\right\}=\mathbb{E}\left\{\left|h_{22}\right|^{2}\right\}=1$, we analyze the performance of the studied approaches with respect to the spectral efficiency constraints assuming $S_{1}^{\min }=S_{2}^{\min }$. If, for a given instance, two links happen to have same exact instantaneous value, we assume for the sake of our approximations that the direct one is larger than the indirect one.

For the transmit power-dominated regime, our simulations show that when the difference between indirect and direct channels grows, so does the energy efficiency, as can be seen in Fig. 2. It can also be seen that a small value of $R_{1}$ has a positive impact for both TDMA and INTF (since both direct channels are larger than both indirect channels). This effect decreases with $R_{1}$. In fact, when $\log _{2} R_{1}=0$ (all equal links), the energy efficiency of both DIV and DRCP is larger than that of TDMA and INTF, and for $\log _{2} R_{1}=3$, the increase is more than double. In this context, both DIV and DRCP have a similar energy efficiency with a slightly larger energy efficiency by DIV. The success rate of this scenario also shows that DIV, DRCP, and TDMA have a good performance as seen in Fig. 3 although with a decline of DRCP for high values of $\log _{2} R_{1}$. The spectral efficiency results are not shown since

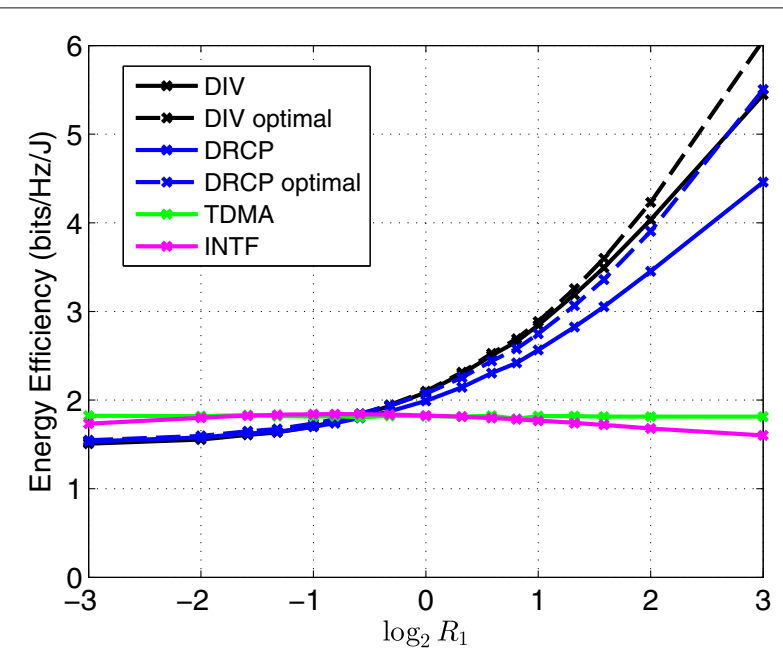

Fig. 2 Energy efficiency of the studied approaches in the transmit power-dominated regime with $S_{1}^{\min }=S_{1}^{\min }=0.3 \mathrm{bits} / \mathrm{Hz}$

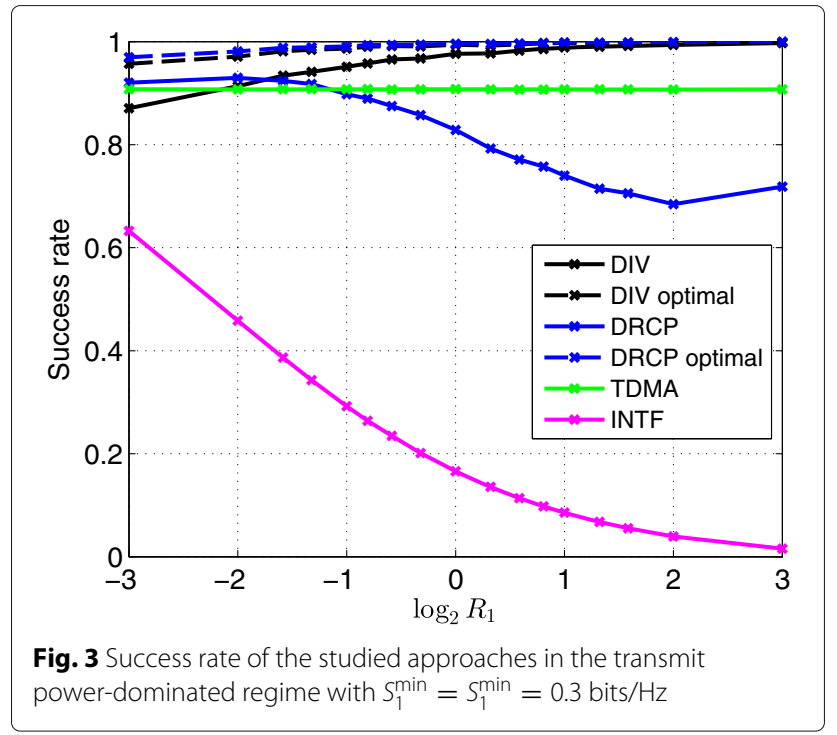

optimizing the energy efficiency in the transmit powerdominated regime boils down to using the transmit power that satisfies the minimum spectral efficiency constraints.

On the other hand, when one indirect channel grows while the other decreases, we can still see that both DIV and DRCP have the best performance for any value of $\log _{2} R_{2}$ as seen in Fig. 4. This shows that both DIV and DRCP are able to exploit one or both of the indirect channels to achieve a higher energy efficiency. In terms of success rate, the performance of DIV, DRCP, and TDMA is higher than 0.8 as seen in Fig. 5. This highlights the result that when at least one indirect link is larger than the direct links, exploiting the overhearing capabilities of the TXs provides the highest performance.

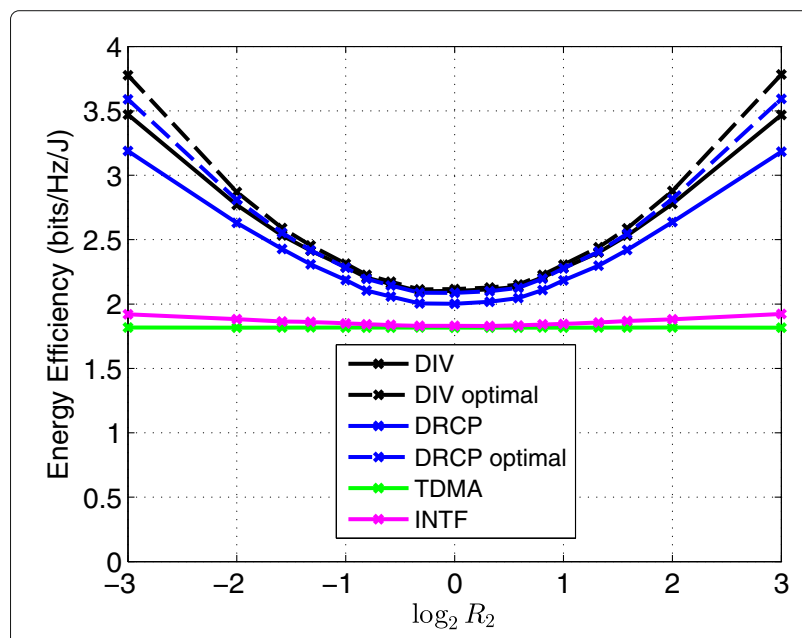

Fig. 4 Energy efficiency of the studied approaches in the transmit power-dominated regime with $S_{1}^{\min }=S_{1}^{\min }=0.3 \mathrm{bits} / \mathrm{Hz}$ 


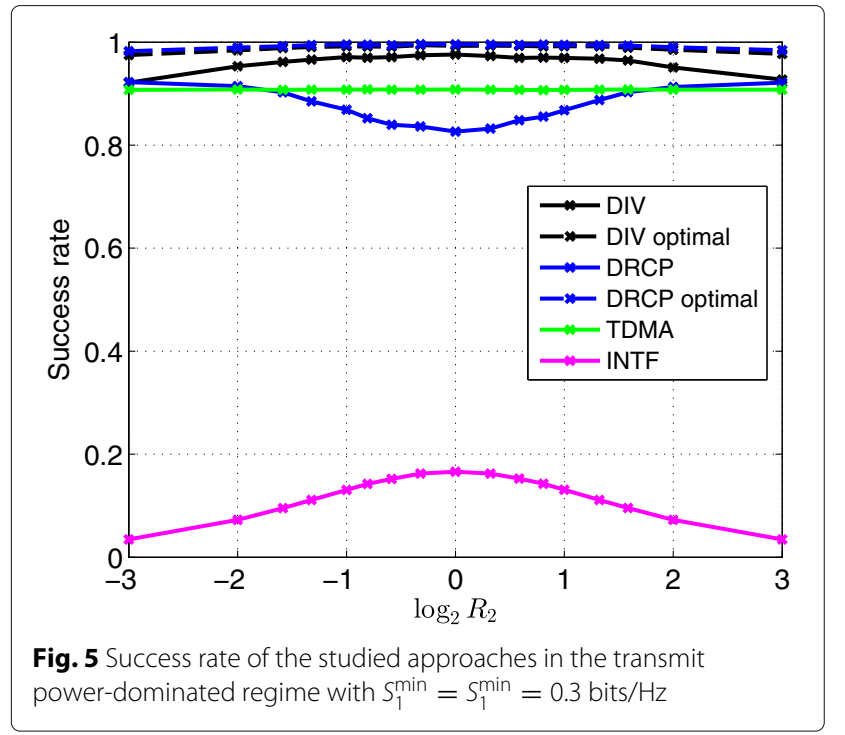

For the circuit power-dominated regime, our simulations show a similar trend where the energy efficiency of DIV and DRCP increase with $\log _{2} R_{1}$ as seen in Fig. 6. However, only above a value of $\log _{2} R_{1}=1.5$ and $\log _{2} R_{1}=2.5$ does DIV and DRCP show a higher energy efficiency than INTF and TDMA, respectively. Since here we mainly consider maximum transmit powers, it is interesting to see a similar behavior in the spectral efficiency as seen in Fig. 7. However, the spectral efficiency of INTF turns out to be the highest due to the pre-log factor and the low transmit power conditions. This comes at the price of a very low success rate as can be seen in Fig. 8.

Finally, it is interesting to notice that the energy efficiency also depends on the spectral efficiency constraints. Considering all equal links, we can see in Figs. 9 and 10

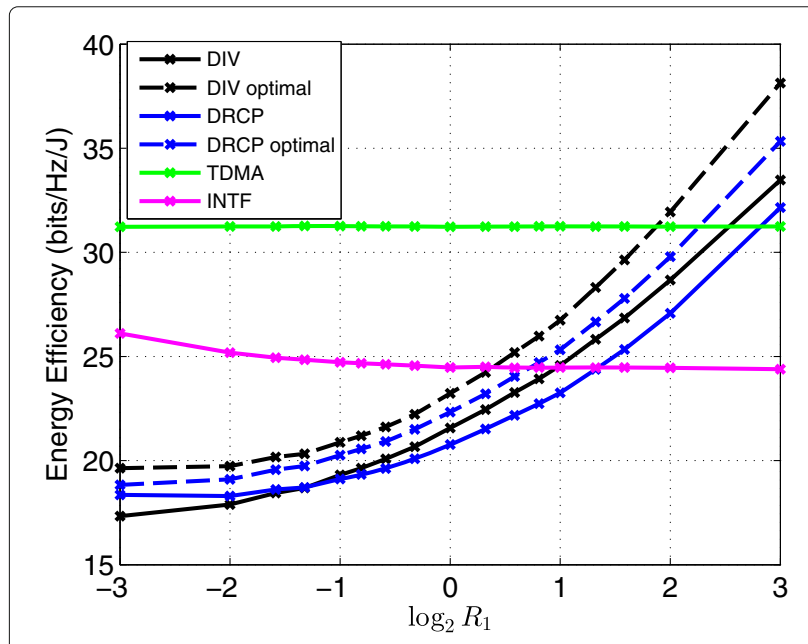

Fig. 6 Energy efficiency of the studied approaches in the circuit power-dominated regime with $S_{1}^{\min }=S_{1}^{\min }=0.3 \mathrm{bits} / \mathrm{Hz}$

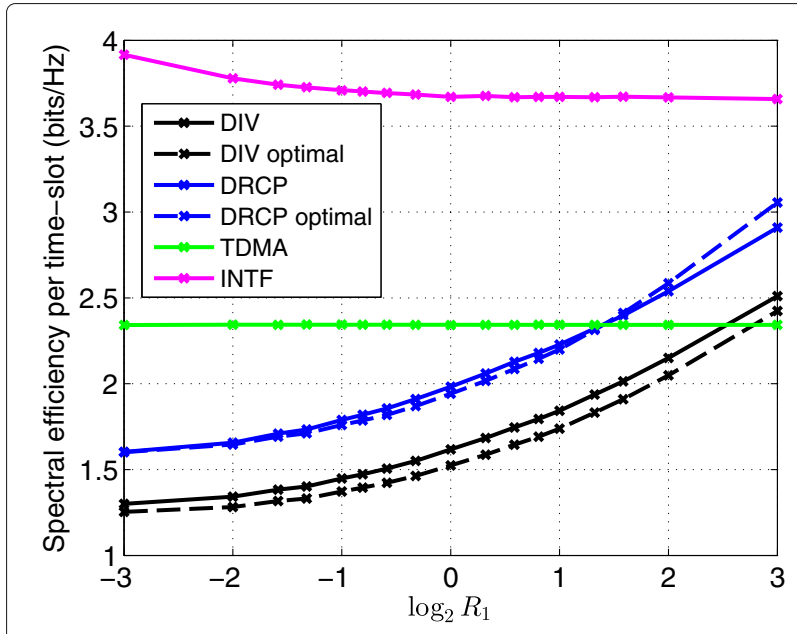

Fig. 7 Spectral efficiency of the studied approaches in the circuit power-dominated regime with $S_{1}^{\min }=S_{1}^{\min }=0.3 \mathrm{bits} / \mathrm{Hz}$

for the transmit power-dominated regime that DIV and DRCP achieve the highest energy efficiency and success rate when the minimum spectral efficiencies are small. However, when the minimum spectral efficiencies are large, a large difference between indirect and direct links is necessary to provide a similar performance. Again, considering all equal channel gains, we observe in Figs. 11 and 12 that TDMA has the largest energy efficiency, but DRCP and DIV have the largest success rate. This shows that strong indirect links are necessary in this regime for the overhearing strategies to achieve a higher energy efficiency.

The choice of whether to use DIV or DRCP depends on the spectral efficiency constraints. In the transmit powerdominated regime, DIV has a higher energy efficiency

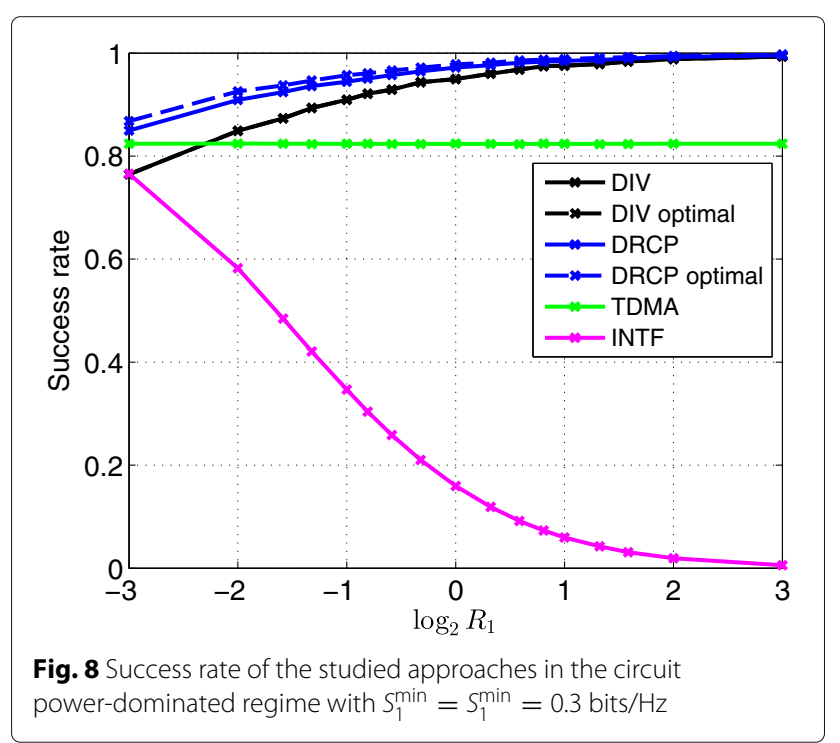




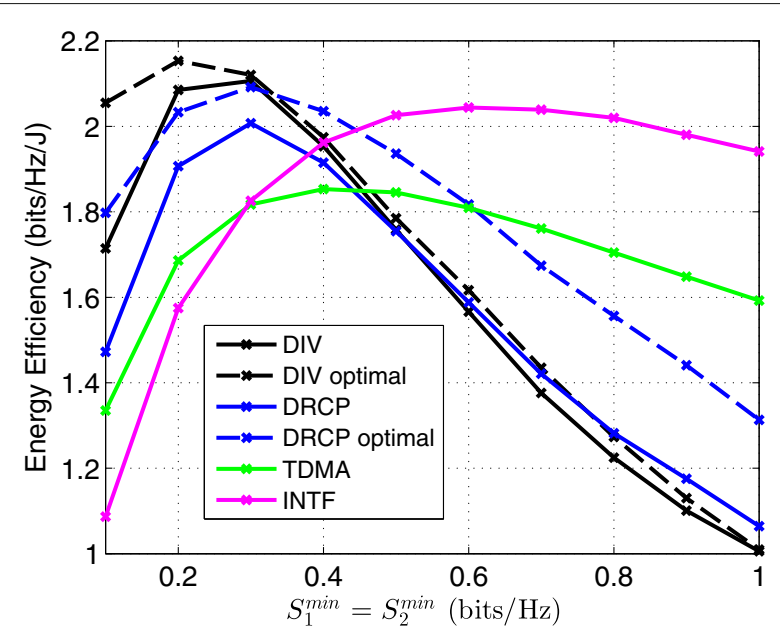

Fig. 9 Energy efficiency of the studied approaches in the transmit power-dominated regime with equal average links

than DRCP when the minimum spectral efficiencies are small, while DRCP has a higher energy efficiency when the minimum spectral efficiencies are large. This is because DIV requires more time slots per transmission round, and hence a higher transmit power, especially when the spectral efficiency constraint is high. In the circuit powerdominated regime, the transmit power used is maximum or binary; hence, the number of time slots are not considered. Therefore, DIV shows a higher energy efficiency for any spectral efficiency constraints. However, in both cases, the success rate for DIV drops faster than that for DRCP.

\section{Conclusions}

In this paper, we have analyzed two approaches that exploit the overhearing capabilities of TXs in terms of

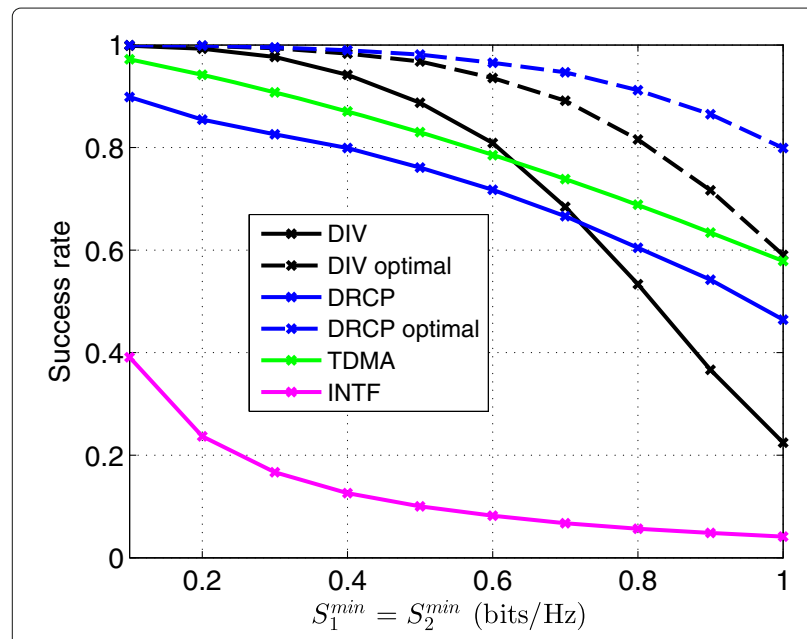

Fig. 10 Success rate of the studied approaches in the transmit power-dominated regime with equal average links

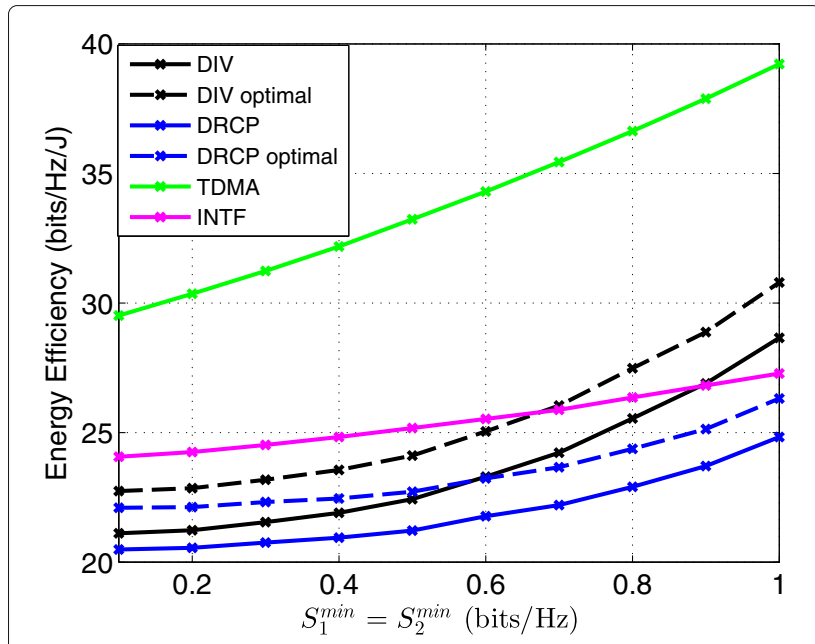

Fig. 11 Energy efficiency of the studied approaches in the circuit power-dominated regime with equal average links

spectral efficiency, energy efficiency, and success rate, under the assumption of a strong overhearing link typical of dense networks. We have also provided a lowcomplexity power control strategy that achieves a performance close to the optimal for each of these approaches.

When comparing the use of overhearing capabilities with other baseline approaches that do not exploit overhearing capabilities, we show that when at least one indirect link is stronger than the direct links, exploiting the overhearing capabilities of the TXs provides the highest performance. In particular, using the transmit power that satisfies the minimum spectral efficiency constraints and using only the strongest links is shown to achieve the best performance in the transmit powerdominated regime, while using maximum transmit power or a binary power with the strongest links achieves the

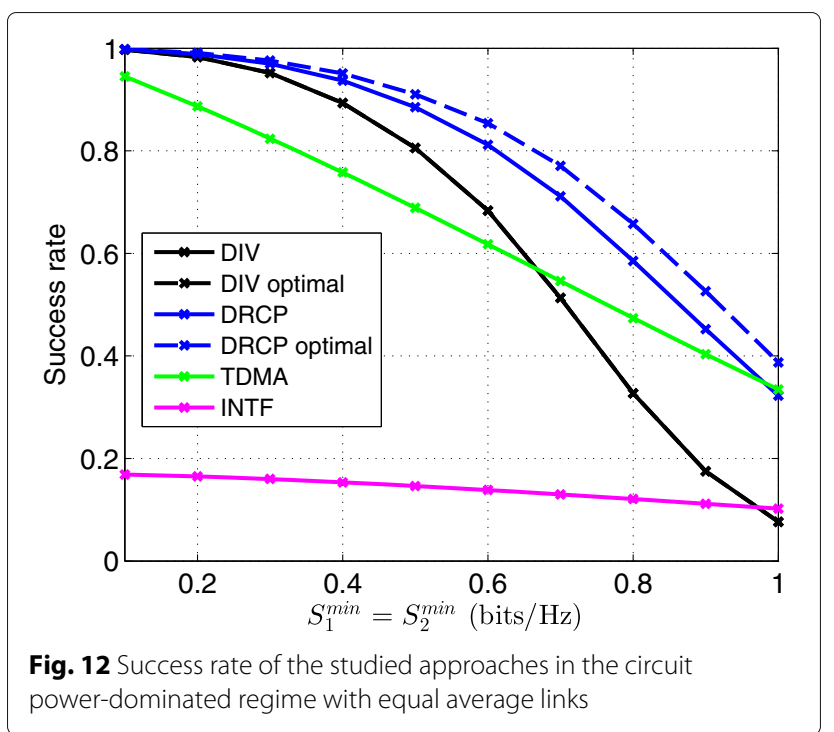


best performance in the circuit power-dominated regime. The choice of using simultaneous transmissions (DRCP) or dedicated time slots (DIV) during the relaying phase depends on the spectral efficiency constraints. Using dedicated time slots shows a larger energy efficiency in the circuit power-dominated regime and when the minimum spectral efficiencies are small in the transmit power dominated regime, while using simultaneous transmissions shows a larger energy efficiency in the transmit powerdominated regime when the minimum spectral efficiencies are large.

\section{Endnotes}

${ }^{1}$ Equation (2) shows the transmission of one symbol by both TXs. However, for some approaches, the symbol will only be transmitted by one TX, leaving Eq. (2) with only half of the terms.

${ }^{2}$ This case happens frequently with closed access base stations where users have a strong interference from a base station that they are not allowed to access and it is one of the main scenarios analyzed by the Small Cell Forum [31]. The analysis presented in this paper can serve as incentive to cooperate with neighboring base stations with the reward of improving the energy efficiency while sharing the same bandwidth.

\section{Appendix A}

We consider the maximization of the following function of $P_{l}^{(t)}$

$$
f\left(P_{l}^{(t)}\right)=\frac{\log \left(1+a P_{l}^{(t)}+b\right)}{k+P_{l}^{(t)}},
$$

where $a>0$ and $P_{l}^{(t)}, b, k \geq 0$. We begin by defining the derivative

$$
\begin{aligned}
& \frac{\partial f\left(P_{l}^{(t)}\right)}{\partial P_{l}^{(t)}} \\
& =\frac{a\left(k+P_{l}^{(t)}\right)-(1+b) \log \left(1+a P_{l}^{(t)}+b\right)-a P_{l}^{(t)} \log \left(1+a P_{l}^{(t)}+b\right)}{\left(1+a P_{l}^{(t)}+b\right)\left(k+P_{l}^{(t)}\right)^{2}} .
\end{aligned}
$$

The derivative of the numerator of (41) is

$$
\frac{\partial g\left(P_{l}^{(t)}\right)}{\partial P_{l}^{(t)}}=-a \log \left(1+a P_{l}^{(t)}+b\right)<0
$$

If $k=b=0$, then $g(0)=0$ and $f\left(P_{l}^{(t)}\right)$ is strictly decreasing with $P_{l}^{(t)}$. Otherwise, $f\left(P_{l}^{(t)}\right)$ has a maximum value found by solving

$$
P_{l}^{(t)}=\frac{e^{W\left(a k+a P_{l}^{(t)}\right)}-1-b}{a},
$$

where $W(x)$ is the Lambert function of $x$. For $P_{l}^{(t)}>0$, Eq. (43) must satisfy $e^{W\left(a k+a P_{l}^{(t)}\right)} \geq 1+b$.

\section{Appendix B}

In this section, we derive a simpler expression for the SNR given by Eq. (24)

$$
\mathrm{SNR}_{1}=\mathbf{a}_{1}^{H} \mathbf{R}_{w_{1}}^{-1} \mathbf{a}_{1} \sigma_{\mathrm{s}}^{2},
$$

where

$$
\mathbf{R}_{w_{1}}=\mathbb{E}\left\{\left(\mathbf{a}_{2} s_{2}+\mathbf{n}_{1}\right)\left(\mathbf{a}_{2} s_{2}+\mathbf{n}_{1}\right)^{H}\right\}=\mathbf{a}_{2} \mathbf{a}_{2}^{H} \sigma_{\mathrm{s}}^{2}+\mathbf{N}_{1},
$$

and
$\operatorname{diag}\left[\sigma_{\mathrm{n}}^{2}, \sigma_{\mathrm{n}}^{2}, \sigma_{\mathrm{n}}^{2}\left(1+\frac{P_{1}^{(3)} \mathbb{E}\left\{\left|h_{11}\right|^{2}\right\}}{P_{2}^{(2)} \mathbb{E}\left\{\left|g_{21}\right|^{2}\right\}}+\frac{P_{2}^{(3)} \mathbb{E}\left\{\left|h_{21}\right|^{2}\right\}}{P_{1}^{(1)} \mathbb{E}\left\{\left|g_{12}\right|^{2}\right\}}\right)\right]$. Using the Sherman-Morrison-Woodbury formula,

$$
\left(\mathbf{A}-\mathbf{B D}^{-1} \mathbf{C}\right)^{-1}=\mathbf{A}^{-1}+\mathbf{A}^{-1} \mathbf{B}\left(\mathbf{D}-\mathbf{C A}^{-1} \mathbf{B}\right)^{-1} \mathbf{C A}^{-1},
$$

where $\mathbf{A}, \mathbf{B}, \mathbf{C}$, and $\mathbf{D}$ are arbitrary matrices, and substituting the following matrices,

$$
\begin{aligned}
& \mathbf{A}=\mathbf{N}_{1}, \mathbf{B}=\mathbf{a}_{2}, \mathbf{C}=\mathbf{a}_{2}^{H} \sigma_{\mathrm{s}}^{2}, \mathbf{D}=-1 \\
& \mathbf{R}_{w_{1}}^{-1} \text { results in } \\
& \quad \mathbf{R}_{w_{1}}^{-1}=\mathbf{N}_{1}^{-1}-\sigma_{\mathrm{s}}^{2} \mathbf{N}_{1}^{-1}\left(1+\sigma_{\mathrm{s}}^{2} \mathbf{a}_{2}^{H} \mathbf{N}_{1}^{-1} \mathbf{a}_{2}\right)^{-1} \mathbf{a}_{2} \mathbf{a}_{2}^{H} \mathbf{N}_{1}^{-1}
\end{aligned}
$$

Now, substituting $\mathbf{R}_{w_{1}}^{-1}$ in Eq. (44) leads to

$$
\begin{aligned}
\mathrm{SNR}_{1} & =\mathbf{a}_{1}^{H}\left(\mathbf{N}_{1}^{-1}-\sigma_{\mathrm{s}}^{2} \mathbf{N}_{1}^{-1}\left(1+\sigma_{\mathrm{s}}^{2} \mathbf{a}_{2}^{H} \mathbf{N}_{1}^{-1} \mathbf{a}_{2}\right)^{-1} \mathbf{a}_{2} \mathbf{a}_{2}^{H} \mathbf{N}_{1}^{-1}\right) \mathbf{a}_{1} \sigma_{\mathrm{s}}^{2} \\
& =\sigma_{\mathrm{s}}^{2} \mathbf{a}_{1}^{H} \mathbf{N}_{1}^{-1} \mathbf{a}_{1}-\sigma_{\mathrm{s}}^{2}\left(1+\sigma_{\mathrm{s}}^{2} \mathbf{a}_{2}^{H} \mathbf{N}_{1}^{-1} \mathbf{a}_{2}\right)^{-1}\left|\mathbf{a}_{2}^{H} \mathbf{N}_{1}^{-1} \mathbf{a}_{1}\right|^{2} \sigma_{\mathrm{s}}^{2} .
\end{aligned}
$$

Using the definition of vectors $\mathbf{a}_{1}$ and $\mathbf{a}_{2}$ leads to

$$
\begin{aligned}
\mathbf{a}_{1}^{H} \mathbf{N}_{1}^{-1} \mathbf{a}_{1} & =\frac{P_{1}^{(1)}\left|h_{11}\right|^{2}}{\sigma_{\mathrm{n}}^{2}}+\frac{P_{2}^{(3)}\left|h_{21}\right|^{2}}{\sigma_{\mathrm{n}}^{2}\left(1+\frac{\sqrt{P_{1}^{(3)}} h_{11}}{\sqrt{P_{2}^{(2)}} g_{21}}+\frac{\sqrt{P_{2}^{(3)}} h_{21}}{\sqrt{P_{1}^{(1)}} g_{12}}\right)} \\
\left|\mathbf{a}_{2}^{H} \mathbf{N}_{1}^{-1} \mathbf{a}_{1}\right|^{2} & =\frac{P_{1}^{(3)} P_{2}^{(3)}\left|h_{11}\right|^{2}\left|h_{21}\right|^{2}}{\sigma_{\mathrm{n}}^{4}\left(1+\frac{\sqrt{P_{1}^{(3)}} h_{11}}{\sqrt{P_{2}^{(2)}} g_{21}}+\frac{\sqrt{P_{2}^{(3)}} h_{21}}{\sqrt{P_{1}^{(1)}} g_{12}}\right)^{2}} \\
\mathbf{a}_{2}^{H} \mathbf{N}_{1}^{-1} \mathbf{a}_{2} & =\frac{P_{2}^{(2)}\left|h_{21}\right|^{2}}{\sigma_{\mathrm{n}}^{2}}+\frac{P_{1}^{(3)}\left|h_{11}\right|^{2}}{\sigma_{\mathrm{n}}^{2}\left(1+\frac{\sqrt{P_{1}^{(3)}} h_{11}}{\sqrt{P_{2}^{(2)}} g_{21}}+\frac{\sqrt{P_{2}^{(3)}} h_{21}}{\sqrt{P_{1}^{(1)}} g_{12}}\right)} .
\end{aligned}
$$


Substituting Eqs. (49) in (48) leads to

$$
\begin{aligned}
& \mathrm{SNR}_{1}=\sigma_{\mathrm{s}}^{2}\left(\frac{P_{1}^{(1)}\left|h_{11}\right|^{2}}{\sigma_{\mathrm{n}}^{2}}+\frac{P_{2}^{(3)}\left|h_{21}\right|^{2}}{\sigma_{\mathrm{n}}^{2}\left(1+\frac{\sqrt{P_{1}^{(3)}} h_{11}}{\sqrt{P_{2}^{(2)}} g_{21}}+\frac{\sqrt{P_{2}^{(3)}} h_{21}}{\sqrt{P_{1}^{(1)}} g_{12}}\right)}\right) \\
& -\left(1+\sigma_{\mathrm{s}}^{2}\left(\frac{P_{2}^{(2)}\left|h_{21}\right|^{2}}{\sigma_{\mathrm{n}}^{2}}+\frac{P_{1}^{(3)}\left|h_{11}\right|^{2}}{\sigma_{\mathrm{n}}^{2}\left(1+\frac{\sqrt{P_{1}^{(3)}} h_{11}}{\sqrt{P_{2}^{(2)}} g_{21}}+\frac{\sqrt{P_{2}^{(3)}} h_{21}}{\sqrt{P_{1}^{(1)}} g_{12}}\right)}\right)\right)^{-1} \\
& \times \frac{\sigma_{\mathrm{s}}^{4} P_{1}^{(3)} P_{2}^{(3)}\left|h_{11}\right|^{2}\left|h_{21}\right|^{2}}{\sigma_{\mathrm{n}}^{4}\left(1+\frac{\sqrt{P_{1}^{(3)}} h_{11}}{\sqrt{P_{2}^{(2)}} g_{21}}+\frac{\sqrt{P_{2}^{(3)}} h_{21}}{\sqrt{P_{1}^{(1)}} g_{12}}\right)^{2}} \\
& =\frac{\sigma_{\mathrm{s}}^{2}}{\sigma_{\mathrm{n}}^{2}} P_{1}^{(1)}\left|h_{11}\right|^{2}+\frac{\sigma_{\mathrm{s}}^{2}}{\sigma_{\mathrm{n}}^{2}} P_{2}^{(3)}\left|h_{21}\right|^{2} \\
& \times\left(\frac{1+\frac{\sigma_{5}^{2}}{\sigma_{n}^{2}} P_{2}^{(2)}\left|h_{21}\right|^{2}}{\frac{\sigma_{s}^{2}}{\sigma_{n}^{2}} P_{1}^{(3)}\left|h_{11}\right|^{2}+\left(\frac{\sigma_{s}^{2}}{\sigma_{n}^{2}} P_{2}^{(2)}\left|h_{21}\right|^{2}+1\right)\left(\frac{\sqrt{P_{1}^{(3)}} h_{11}}{\sqrt{P_{2}^{(2)}} g_{21}}+\frac{\sqrt{P_{2}^{(3)}} h_{21}}{\sqrt{P_{1}^{(1)}} g_{12}}+1\right)}\right) \\
& =\gamma_{11}^{(1)}+\frac{\gamma_{21}^{(3)}}{\frac{\gamma_{11}^{(3)}}{\gamma_{21}^{(2)}+1}+\frac{\gamma_{11}^{(3)}}{\xi_{21}^{(2)}}+\frac{\gamma_{21}^{(3)}}{\xi_{12}^{(1)}}+1} \text {. }
\end{aligned}
$$

Following an analogous derivation, $\mathrm{SNR}_{2}$ is given by

$$
\begin{aligned}
\mathrm{SNR}_{2}= & \frac{\sigma_{\mathrm{s}}^{2}}{\sigma_{\mathrm{n}}^{2}} P_{2}^{(2)}\left|h_{22}\right|^{2}+\frac{\sigma_{\mathrm{s}}^{2}}{\sigma_{\mathrm{n}}^{2}} P_{1}^{(3)}\left|h_{12}\right|^{2} \\
& \times\left(\frac{1+\frac{\sigma_{\mathrm{s}}^{2}}{\sigma_{\mathrm{n}}^{2}} P_{1}^{(1)}\left|h_{12}\right|^{2}}{\frac{\sigma_{\mathrm{s}}^{2}}{\sigma_{\mathrm{n}}^{2}} P_{2}^{(3)}\left|h_{22}\right|^{2}+\left(\frac{\sigma_{\mathrm{s}}^{2}}{\sigma_{\mathrm{n}}^{2}} P_{1}^{(1)}\left|h_{12}\right|^{2}+1\right)\left(\frac{\sqrt{P_{1}^{(3)}} h_{12}}{\sqrt{P_{2}^{(2)}} g_{21}}+\frac{\sqrt{P_{2}^{(3)}} h_{22}}{\sqrt{P_{1}^{(1)}} g_{12}}+1\right)}\right) \\
= & \gamma_{22}^{(2)}+\frac{\gamma_{12}^{(3)}}{\frac{\gamma_{22}^{(3)}}{\gamma_{12}^{(1)}+1}+\frac{\gamma_{22}^{(3)}}{\xi_{12}^{(1)}}+\frac{\gamma_{12}^{(3)}}{\xi_{21}^{(2)}}+1} .
\end{aligned}
$$

\section{Acknowledgements}

This research work was carried out at the ESAT Laboratory of KU Leuven, in the frame of KU Leuven Research Council PFV/10/002 (OPTEC), FWO project G091213N "Cross-layer optimization with real-time adaptive dynamic spectrum management for fourth generation broadband access networks," and the Belgian Programme on Interuniversity Attraction Poles initiated by the Belgian Federal Science Policy Office "Belgian network on stochastic modelling, analysis, design and optimization of communication systems (BESTCOM)" 2012-2017.

\section{Competing interests}

The authors declare that they have no competing interests.

\section{Publisher's Note}

Springer Nature remains neutral with regard to jurisdictional claims in published maps and institutional affiliations.

\section{Author details}

${ }^{1}$ STADIUS Center for Dynamical Systems, Signal Processing and Data Analytics, Department of Electrical Engineering (ESAT), KU Leuven, Kasteelpark Arenberg 10, 3000 Leuven, Belgium. ${ }^{2}$ MICAS, Microelectronics and Sensors, Department of Electrical Engineering (ESAT), KU Leuven, Kasteelpark Arenberg 10, 3000 Leuven, Belgium. ${ }^{3}$ TELEMIC, Telecommunications and Microwaves, Department of Electrical Engineering (ESAT), KU Leuven, Kasteelpark Arenberg 10, 3000 Leuven, Belgium. ${ }^{4}$ Université Catholique de Louvain (UCL), ICTEAM
Institute, Digital Communications Group, Place du Levant 2, 1348 Louvain-la-Neuve, Belgium.

Received: 15 March 2017 Accepted: 21 October 2017

Published online: 06 November 2017

\section{References}

1. CX Wang, F Haider, X Gao, XH You, Y Yang, D Yuan, HM Aggoune, H Haas, S Fletcher, E Hepsaydir, Celluar architecture and key technologies for $5 \mathrm{G}$ wireless communication networks. IEEE Commun. Mag. 52(2), 122-130 (2014)

2. N Bushan, J Li, D Malladi, R Gilmore, D Brenner, A Damnjanovic, RT Sukhavasi, C Patel, S Geirhofer, Network densification: the dominant theme for wireless evolution into 5G. IEEE Commun. Mag. 52(1), 82-89 (2014)

3. M Kamel, W Hamouda, A Youssef, Ultra-dense networks: a survey. IEEE Commun. Surv. Tutor. 18(4), 2522-2545 (2016)

4. SF Yunas, M Valkama, J Niemela, Spectral and energy efficiency of ultra-dense networks under different deployment strategies. IEEE Commun. Mag. 53(1), 90-100 (2015)

5. Z Sheng, J Fan, CH Liu, VCM Leung, X Liu, KK Leung, Energy-efficient relay selection for cooperative relaying in wireless multimedia networks. IEEE Trans. Veh. Technol. 64(3), 1156-1170 (2015)

6. C Sun, C Yang, Energy-efficient analysis of one-way and two-way relay systems. EURASIP J. Wirel. Commun. Netw. 2012(46), 1-18 (2012)

7. S-Y Robert Li, RW Yeung, N Cai, Linear network coding. IEEE Trans. Inf. Theory. 49(2), 371-381 (2003)

8. RHY Louie, Y Li, B Vucetic, Practical physical layer network coding for two-way relay channels: performance analysis and comparison. IEEE Trans. Wirel. Commun. 9(2), 764-777 (2010)

9. H Yang, K Lee, J Chun, Zero-forcing based two-phased relaying. IEEE Int. Conf. Commun. (ICC). (Glasgow, 2007), pp. 5224-5228

10. IJ Baik, SY Chung, Network coding for two-way relay channels using lattices. IEEE Int. Conf. Commun. (ICC). (Beijing, 2008), pp. 3898-3902

11. S Smirani, M Kamoun, M Sarkiss, A Zaidi, P Duhamel, Achievable rate regions for two-way relay channel using nested latice coding. IEEE Trans. Wirel. Commun. 13(10), 5607-5620 (2014)

12. L Fang, J Xu, L Qiu, in IEEE International Conference on Communications (ICC). Energy efficiency optimization for two-way relay channels, (Budapest, 2013)

13. M Zhou, Q Cui, R Jantti, X Tao, Energy-efficient relay selection and power allocation for two-way relay channel with analog network coding. IEEE Commun. Lett. 16(6), 816-819 (2012)

14. C Sun, C Yang, Is two-way relay more energy efficient? IEEE Global Telecommunications Conference (GLOBECOM), (Houston, 2011)

15. CXiong, L Lu, G Ye Li, Energy-efficient OFDMA-based two-way relay. IEEE Trans. Commun. 63(9), 3157-3169 (2015)

16. S Katti, I Maric, A Goldsmith, D Katabi, M Medard, Joint relaying and network coding in wireless networks. IEEE International Symposium on Information Theory (ISIT), (Nice, 2007)

17. G Ye Li, Z Xu, C Xiong, C Yang, S Zhang, Y Chen, S Xu, Energy-efficient wireless communications: tutorial, survey, and open issues. IEEE Wirel. Commun. 18(6), 28-35 (2011)

18. M Nokleby, B Aazhang, User cooperation for energy-efficient cellular communications. IEEE Int. Conf. Commun (2010)

19. A Ghosh, N Mangalvedhe, R Ratasuk, B Mondal, M Cudak, E Visotsky, TA Thomas, JG Andrews, P Xia, HS Ho, HS Dhillon, TD Novlan, Heterogeneous cellular networks: from theory to practice. IEEE Commun. Mag. 50(6), 54-64 (2012)

20. D Feng, C Jiang, G Lim, LJ Cimini Jr., G Feng, G Ye Li, A survey of energy-efficient wireless communications. IEEE Commun. Surv. Tutor. 15(1), 167-178 (2013)

21. V Chandrasekhar, JG Andrews, A Gatherer, Femtocell networks: a survey. IEEE Commun. Mag. 46(9), 59-67 (2008)

22. HQ Lai, KJR Liu, Space time network coding. IEEE Trans. Signal Process. 59(4), 1706-1718 (2011)

23. KXiong, P Fan, HC Yang, KB Letaief, Space time network coding with overhearing relays. IEEE Trans. Signal Process. 13(7), 3567-3582 (2014)

24. Y Zhang, KXiong, P Fan, X Di, X Zhou, Outage performance of space time network coding with overhearing AF relays. IEEE Commun. Lett. 19(12), 2234-2237 (2015) 
25. R Torrea-Duran, F Rosas, ZKZ Khan, S Pollin, P Tsiaflakis, M Moonen, Double relay communication protocol for bandwidth management in cellular systems. Eur. Signal Process. Conf. (EUSIPCO 2015). (Nice, 2015), pp. 2137-2141

26. G Auer, V Giannini, C Desset, I Godor, P Skillermark, M Olsson, M Ali Imran, D Sabella, MJ Gonzalez, O Blume, A Fehske, How much energy is needed to run a wireless network? IEEE Wirel.Commun. 18(5), 40-49 (2011)

27. G Miao, N Himayat, GY Li, AT Koc, S Talwar, in IEEE International Conference on Communications (ICC). Interference-aware energy-efficient power optimization, (Dresden, 2009)

28. C Li, SH Song, J Zhang, KB Letaief, Maximizing energy efficiency in wireless networks with a minimum average throughput requirement. IEEE Wireless Communications and Networking Conference, (Shanghai, 2012), pp. 1130-1134

29. A Gjendemsjo, D Gesbert, GE Oien, SG Kiani, Binary power control for sum rate maximization over multiple interfering links. IEEE Trans. Wirel. Commun. 7(8), 3164-3173 (2008). Shanghai

30. D Tse, P Viswanath, Fundamentals of wireless communication. (Cambridge University Press, 2005)

31. Femto Forum, Interference management in UMTS Femtocells. http://www.femtoforum.org/femto/Files/File/FFuMTS-

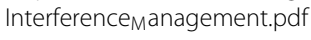

\section{Submit your manuscript to a SpringerOpen ${ }^{\circ}$ journal and benefit from:}

- Convenient online submission

- Rigorous peer review

- Open access: articles freely available online

- High visibility within the field

- Retaining the copyright to your article

Submit your next manuscript at $>$ springeropen.com 